\title{
Вехи панории. Действие, как основа материи, пространства, мира
}

\author{
А. Н. Пан
}

\begin{abstract}
Аннотация
Предлагается описание строения материи и мира на основе изменения действие-длительность. Для его гармоник вводится понятие места и пространственные отношения. Показано, как мир возникает из внепространственного шума и развивается до надшумного пространственного строения. Скрывающаяся за кажущимся пустым пространством среда, имеющая огромную плотность, является причиной электрического и гравитационного полей. Объясняется строение частиц.
\end{abstract}

\section{Введение}

K концу 19 века в физике использовались, кроме абсолютного времени, три основных понятия: тело, поле и пространство. Тела движутся в пространстве, служат источниками полей и через них взаимодействуют с другими телами. Тела и поля находятся в пустом пространстве, которое не материально, но имеет свойства: бесконечность, три измерения и расстояния. Кроме того, начиная с Декарта, предполагалось, что в пространстве имеется ненаблюдаемая среда - эфир [1], в котором должны происходить все явления: поля - волны, а тела - особенности строения или движения (например вихри). Все части материи обладают энергией, которая связана со временем, как способность совершать работу изменять состояние. Следовательно она есть характеристика изменения.

$\mathrm{K}$ началу 20 века опыт, а затем теория, нашли несоответствия и противоречия в этой картине мира [1]. Это привело к созданию двух новых механик: теории относительности $[2,3]$ и квантовой $[4,5]$. В них прежде чёткие понятия тел, волн, полей и пространства стали расплываться, 
пересекаться и взаимодействовать между собой. Понятие эфира было признано ошибочным, т. к. его теории противоречили опыту [1, 2]. Однако его пытались и по-прежнему пытаются возродить.

Продолжение попыток возродить эфир связано с особыми и непривычными характеристиками, которыми новые механики наделяли пустое пространство. Эти характеристики более соответствуют среде, чем пустоте [6]. Притяжение, присущее всем телам, полям и энергиям, меняет "пустоту", которая теперь влияет на движение других материй [3]. "Пустота" представляется "физическим вакуумом" [7] с неустранимыми нулевыми колебаниями, виртуальными частицами и всплесками энергии.

Механики Эйнштейна и квантовая достаточно хороши для описания результатов опытов и наблюдений, но их основания до сих пор остаются неясными. Во многом это связано с индуктивным путём развития физики - от наблюдения обыденных явлений к простым законам для них и дальнейшее последовательное усложнение законов (теорий) по мере расширения опытных знаний, сопровождающееся ростом их отвлечённости. Отвлечённые орудия познания (симметрии, группы, сложные пространства) позволяют увидеть как-бы сверху издали общие закономерности, но не достаточно углубляются в их основы. Однако эти неясные основания оставляют в физических законах свои следы. Это энергия, действие, время, пространство.

Энергия содержится во всём. Являясь характеристикой изменения, она переносит на него свою всеобщность. Тогда можно утверждать, что всё есть изменение. Оно имеет две составляющие (величину, длительность) и не сводится к чему-либо другому. Замена одной величины или длительности на другую лишь переводит одно изменение в другое.

Чтобы иметь возможность сравнивать и тем самым измерять изменение, нужен его образец, с которым можно сравнивать, и способ сравнения. Это даёт меру. Один образец величины берётся за условно постоянный нуль - начало системы отсчёта. Другой - за условную единицу. Переход к другим образцам, возможно не постоянным относительно первых, меняет измеряемое изменение полностью, но оставляет его изменением. Образец длительности, с которым сравнивают другие изменения, определяет время: такое-то изменение соответствует такому-то количеству образцов, или такой-то его части.

Всеобщий мир изменений (мен) сам по себе не имеет каких-либо выделенных величин и длительностей. Но после выбора нуля можно утверждать, что он состоит из стационарных повторов, т. к. только они да- 
ют постоянство перемен. Нуль, как средняя линия повторов, становится единственной сохраняющейся величиной, определяющей пустотность всего. Нестационарность возможна лишь временно, как частный случай.

Бесконечное разнообразие повторов мена удобно и привычно описывать бесконечным набором гармоник любых частот, амплитуд и фаз. Из-за отсутствия каких-либо выделенных величин или длительностей мен масштабно инвариантен. В нём нет особых гармоник со свойствами, отличными от других. Представление мена с помощью гармоник не есть разложение Фурье, которое применимо только к одной определённой периодической функции времени, возможно с бесконечным в пределе периодом. Мен есть бесконечное сочетание множества таких функций. Соответственно требуется не набор гармоник, в котором каждую частоту имеет только одна гармоника, а бесконечное множество гармоник каждой частоты.

В любой гармонике представлен весь мен: Частота и амплитуда задают её масштаб. Другие гармоники с меньшей частотой в сумме составляют её среднюю линию (принимаемую за нуль отсчёта), а с большей - шум колебаний, который в теории вероятности описывается произведением случайной амплитуды на определённое колебание.

Отсюда следуют общие метафизические выводы:

Относительно любой гармоники мен не пуст и переменен. Для каждой гармоники найдётся гармоника, которая колеблется с ней в противофазе и имеет такие же амплитуду и частоту. Поэтому сумма всех гармоник равна нулю. Тогда в целом мен пуст и тем постоянен. Всеобщая пустотность проявляется через частную переменчивую полноту. Всё есть ничто, но является как нечто.

Любая часть мена, какой-бы малой она не казалась, противоположна всему остальному, т. к. их сумма есть пустое всё. Следовательно она по величине равна всему остальному. Любая часть имеет нуль, который есть всё. Тогда часть содержит целое со всеми его частями, включая себя, вместе с их прошлым, настоящим и будущим.

Такими же свойствами обладают в математике бесконечные множества. В них можно выделить любое конечное или бесконечное число подмножеств, изоморфных (тождественных) всему множеству. Каждое из них имеет свои подмножества, изоморфные множеству, а значит содержит его в себе. Можно делить бесконечность на бесконечные части с собственными бесконечными частями, изоморфными начальной бесконечности. Это логика бесконечностей, отличная от обычной логики 
конечного, где часть не превосходит целого.

В любой бесконечной абелевой группе по сложению/умножению с наличием нуля/единицы сумма/произведение всех элементов группы есть нуль/единица, как их отсутствие (кроме нуля/единицы), содержащее все элементы. Вся бесконечная группа суммарно умещается в одном нулевом или единичном элементе. В частности таким свойством обладают разные множества чисел (целых, рациональных, вещественных, комплексных и др.), включающих в себя нуль и/или единицу с симметрией относительно них.

Множество гармоник одной частоты образует абелеву группу по сложению. Она двумерна - две амплитуды (множители перед $\cos \omega t$ и $\sin \omega t$, или амплитуда и начальная фаза). Этой группы достаточно для выражения указанных свойств мена, но недостаточно для его полного описания, требующего трёхмерного семейства групп гармоник всех частот, которое уже не составляет группу.

Бесконечность обуславливает ещё два свойства мена. Т. к. бесконечное множество можно разбить на любое, вплоть до бесконечного, число изоморфных ему подмножеств, то мен разложим на любое количество точно таких же независимых менов - Целое бесконечно делимо на повторы самого себя. Мен в целом пуст, но обладает бесконечной энергией, как бесконечный набор гармоник всех частот и амплитуд. Любая его часть, как содержащая всё, тоже имеет бесконечную энергию. Часть владеет энергией всего.

Мен есть непроявленное состояние (неявь, хаос), из которого проявляются (рождаются) частные проявленные миры (явь, космос). Их множество и разнообразие бесконечно. Найдутся миры с любыми свойствами. Наш мир есть один из бесконечного множества миров. Он временен и переменен. Его особые свойства логически не выводимы полностью из общих соображений, хотя и неразрывно связаны с ними. Наш мир познаётся, отталкиваясь от частного опыта нашей жизни в нашем мире.

В метафизике эти выводы не новы. Но здесь они получены из физических соображений.

\section{Гармоника действие-энергия}

Во введении предложено повторы изменений представлять бесконечным множеством гармоник. Уравнение гармоники и его решение есть 
$\ddot{s} \equiv d^{2} s / d t^{2}=-\Omega^{2} s, \quad s(t, \varphi)=S \cos (\Omega t+\varphi)=R e S e^{i(\Omega t+\varphi)}$.

Колебание $s(t)$ характеризуется частотой $\Omega$ (свойство механической системы, поддерживающей колебание) и амплитудой $S$ (его интенсивность). Начальная фаза $\varphi$ даёт сдвиг колебания во времени. Так две гармоники с одинаковыми $\Omega$ и $S$, но разными $\varphi\left(\varphi_{1}, \varphi_{2}\right)$ представляют собой два совпадающих колебания, которые сдвинуты по фазе на $\varphi_{1}-\varphi_{2}$. В таком же соотношении находятся два одинаковых тела в разных местах пространства. Т. о. начальную фазу можно назвать место гармоники. Движение гармоники в пространстве мест со скоростью $\dot{\varphi}$ соответствует изменению частоты на эту скорость. Другая гармоника с частотой $\Omega_{1}$ движется относительно первой со скоростью $\Omega_{1}-\Omega$.

Введение места возможно лишь при его медленном изменении $\dot{\varphi} \ll \Omega$. Если $\dot{\varphi} \sim \Omega$, то понятие места расплывается - в пределе гармоника за свой период успевает пройти всё пространство мест. Её период становится квантом времени, ограничивающем применение не только временны́х, но и пространственных отношений.

Из $\Omega$ и $S$ составляются ещё две величины, характеризующие гармонику как целое. Это импульс гармоники $\Omega S$, представляющий её вовне, и движущая её внутренняя энергия (внутэн) $\Omega^{2} S^{2}$. В квантовой механике $\Omega \hbar$, где $\hbar$ - квант действия Планка, есть энергия осциллятора, участвующая в обмене. Если так же для гармоники мена считать $\Omega S$ энергией, то $S$ должна быть амплитудой действия, а $s-$ отрицательным действием, чтобы его скорость $e=\dot{s}$ была колеблющейся энергией и соответствовала общепринятой связи энергии с действием. Т.о. импульс гармоники является её внешней энергией (внешэн), участвующая во внешних взаимодействиях. Образуется гармоника "действие-энергия", сокращённо гдэн (Г), а переменное действие становится основой всего существующего.

После выделения любой частоты гдэнов, её можно взять за единицу измерения $\Omega=1$. Это делает время безразмерным $t=\Omega t$ а энергии и действию даёт одинаковые размерности.

$$
s=S_{c} \cos t+S_{s} \sin t=S \cos (t+\varphi)=\operatorname{Re} S e^{i(t+\varphi)},
$$

где $S_{c}=S \cos \varphi, S_{s}=S \sin \varphi$.

Гдэн есть часть мена, выделенная из целого. Выделение части нарушает единство. Для его восстановления требуется между частью Ч и целым Ц вводить связь С: Ц=Ч $+\mathrm{C}+(Ц-Ч-С)$. При этом часть знает 
лишь связь и теряет знание целого, скрывающегося для неё за этой связью. Часть и связь соотносительны - изменение одной сопровождается изменением другой.

Например пружинный маятник. В нём есть колеблющаяся часть (масса, тело) и пружина, связывающая её с остальным неведомым ей миром. Пружинная связь определяет все свойства колебания и является его двигателем. Также гдэн подобно пружинному (или другому) маятнику есть изменение, поддерживаемое извне через связь, которая есть двигатель гдэна, сокращённо двиг.

Сдвинутый на полоборота гдэн с местом $\varphi \pm \pi$ определяется как противогдэн (П). В месте $\varphi$ он представляется как гдэн с отрицательной амплитудой $(-S)$. С введением противогдэнов пространство мест делится на собственно пространство $|\varphi| \leq \pi / 2$ и зеркальное противопространство $\pi / 2 \leq|\varphi| \leq \pi$, в котором Г и П взаимно заменяются. Эти понятия относительны для каждого гдэна. Все гдэны с местами, отличными от его места менее, чем на $\pi / 2$, входят в его пространство. Остальные - в противо-пространство. Сумма $П+Г$ даёт нулевое колебание. Но сами Г и П при этом не исчезают, а скрываются, сохраняя свою энергию.

\section{Шум и надшумные гдэны}

О всеобщем мире изменений больше нечего сообщить, кроме уже приведённого во введении. Чтобы иметь возможность делать более содержательные утверждения требуется вводить ограничения, выделяющие из целого некоторую его часть. Первым ограничением становится выбор бесконечного множества гармоник одной частоты. Для них гармоники с меньшей частотой описывают их переменную среднюю линию, а с бо́льшей - их модуляцию. Средняя линия колебаний гармоник берётся за начало отсчёта - становится нулём, а модуляции надо учитывать особо.

Колебание можно представить как случайную величину (слувел) с вероятностями, равными отношению времени пребывания этой величины к периоду колебания. Тогда к сумме гармоник модуляции применима центральная предельная теорема Ляпунова [8], согласно которой бесконечная сумма слувел с любыми вероятностными распределениями сходится к распределению Гаусса. Модуляция становится равновесными флуктуациями (шумом) на выбранной частоте. 
Величина модуляции может быть любой. Но распределение Гаусса бесконечно делимо $[9,10]$ и представимо как сумма любого числа независимых равновесных слувел. Тогда бесконечная неопределённость величины модуляции заменяется бесконечным множеством шумов с любыми конечными дисперсиями. Один из них является носителем нашего мира. Дисперсия $\left\langle S^{2}\right\rangle$ задаёт масштаб шума, который выражается среднеквадратичным отклонением $\left\langle S^{2}\right\rangle^{1 / 2}$. Его не с чем сравнивать, но можно взять за единицу измерения амплитуд гдэнов $\left\langle S^{2}\right\rangle^{1 / 2}=1$. Т. о. из бесконечного, с нашей точки зрения, всеобщего мира изменений берётся его бесконечно малая часть, которая для нас остаётся по-прежнему бесконечно большой.

Стационарный слувел должен иметь каноническое разложение по гармоникам $[9,10]$. В нём амплитуды $S_{c}$ и $S_{s}$ гармоник $(1)$ есть слувелы с нулевыми средними $\left\langle S_{c}\right\rangle=\left\langle S_{s}\right\rangle=0$ и одинаковыми распределениями Гаусса. Из единичности дисперсии шума следует $\left\langle S^{2}\right\rangle=\left\langle S_{c}^{2}\right\rangle+\left\langle S_{s}^{2}\right\rangle=1$, или $\left\langle S_{c}^{2}\right\rangle=\left\langle S_{s}^{2}\right\rangle=1 / 2$. Тогда плотность вероятности в шуме

$$
P^{e}\left(S_{c}, S_{s}\right)=(1 / \pi) e^{-S^{2}}, \quad S^{2}=S_{c}^{2}+S_{s}^{2} .
$$

Переходя к распределению по $S, \varphi: P^{e}(S, \varphi) d S d \varphi=P^{e}\left(S_{c}, S_{s}\right) d S_{c} d S_{s}$ при $S>0$, получим

$$
P^{e}(S, \varphi)=(S / \pi) e^{-S^{2}}, \quad|\varphi| \leq \pi
$$

Это распределение равномерно по месту $\varphi$. В одномерном шуме нет пространственных отношений.

Первое пространственное свойство появляется при переходе от одномерного шума к многомерному, используя свойство бесконечной делимости распределения Гаусса. Для получения трёхмерного шума слувел $\mathrm{S}$ разбивается на сумму трёх независимых одинаковых слувелов $S_{j}$ с нулевыми средними: $S=\sum_{j=1}^{3} S_{j},\left\langle S^{2}\right\rangle=3\left\langle S_{j}^{2}\right\rangle,\left\langle S_{j}\right\rangle=0$. Если оставить для шума каждого измерения дисперсию $\left\langle S_{j}^{2}\right\rangle=1$, то распределение трёхмерного шума

$$
P^{e}(\mathbf{S}, \varphi)=\prod_{j=1}^{3} P_{j}^{e}\left(S_{j}, \varphi_{j}\right), \quad P_{j}^{e}\left(S_{j}, \varphi_{j}\right)=\left(S_{j} / \pi\right) e^{-S_{j}^{2}} .
$$

Здесь введены векторы $\mathbf{S}=\left\{S_{j}\right\}, \boldsymbol{\varphi}=\left\{\varphi_{j}\right\}, S_{j} \geq 0,\left|\varphi_{j}\right| \leq \pi$.

Одного шума недостаточно, чтобы образовался и существовал сложный и упорядоченный мир. Мир - надшумное строение, состоящее из надшумных гдэнов, которые должны быть способны к объединению 
в более сложные многоуровневые и развивающиеся структуры. Они могут появиться из шума, если его уровень понизится, а флуктуации не успеют к нему подстроиться и сохранятся как надшумные гдэны. Скорость релаксации становится определяющим параметром существования и свойств мира. Её время даёт верхний предел времени его жизни.

Если релаксация велика, то существует только шум, в котором не может быть мира. Если релаксация мала, то при уменьшении шума его большие флуктуации становятся надшумными гдэнами. Т. к. амплитуды гдэнов отсчитываются в единицах уровня шума, то это выглядит как их увеличение - выделение надшумной энергии, которая становится энергией мира. Это есть рождение (проявление) мира из шума (хаоса, неяви и т. п.). Постоянство шума обеспечивает развитие мира с сохранением в нём энергии. Увеличение шума выглядит как уменьшение амплитуд гдэнов и поглощение надшумной энергии мира. Мир погружается (возвращается) в хаос.

Пусть имеются трёхмерные гдэн и шум. Амплитуды колебаний гдэна $S_{c j}$ и $S_{s j}$ берутся из (1), а распределения амплитуд шума - из (2). Их совместное распределение, имеющее средние амплитуды $\overline{S_{c j}}, \overline{S_{s j}}$ и дисперсию шума, образует распределение надшумного гдэна.

$$
P^{\Gamma}\left(\mathbf{S}_{c}, \mathbf{S}_{s}\right)=\prod_{j=1}^{3} P_{j}^{\Gamma}\left(S_{c j}, S_{s j}\right), \quad P_{j}^{\Gamma}\left(S_{c j}, S_{s j}\right)=(1 / \pi) e^{-\stackrel{\circ}{2}_{j}^{2}}
$$

Здесь $\mathbf{S}_{c}=\left\{S_{c j}\right\}, \mathbf{S}_{s}=\left\{S_{s j}\right\}-$ случайные векторы, $\stackrel{\circ}{j}_{j}^{2}=\stackrel{\circ}{S}_{c j}^{2}+\stackrel{\circ}{S}_{s j}^{2}$, $S_{c j}=S_{c j}-\overline{S_{c j}}, S_{s j}=S_{s j}-\overline{S_{s j}}$ - центрированные слувелы (флуктуации). Для этого распределения условие стационарности слувел, которое совпадает с условием канонического разложения (нулевые средние и одинаковые дисперсии), не выполняется. Следовательно распределения надшумных гдэнов нестационарны.

Чтобы найти пространственное распределение надо перейти к переменным $S_{j}$ и $\varphi_{j}$, как при выводе $(3): P_{j}^{\Gamma}\left(S_{j}, \varphi_{j}\right)=S_{j} P_{j}^{\Gamma}\left(S_{c j}, S_{s j}\right)$, $S_{c j}=S_{j} \cos \varphi_{j}, S_{s j}=S_{j} \sin \varphi_{j}$ $P_{j}^{\Gamma}\left(S_{j}, \varphi_{j}\right)=\left(S_{j} / \pi\right) \exp \left\{-\left(S_{j} \cos \varphi_{j}-\overline{S_{c j}}\right)^{2}-\left(S_{j} \sin \varphi_{j}-\overline{S_{s j}}\right)^{2}\right\}$. Вводится $\bar{S}_{j}^{2}=\bar{S}_{c j}^{2}+\bar{S}_{s j}^{2}, \bar{S}_{c j}=\bar{S}_{j} \cos \bar{\varphi}_{j}, \bar{S}_{s j}=\bar{S}_{j} \sin \bar{\varphi}_{j}$, $\bar{\varphi}_{j}=\operatorname{arctg}\left(\bar{S}_{s j} / \bar{S}_{c j}\right), \stackrel{\circ}{\varphi}_{j}=\varphi_{j}-\bar{\varphi}_{j}, \stackrel{\circ}{S}_{j}=S_{j}-\bar{S}_{j},\left\langle S_{j}\right\rangle=\bar{S}_{j} \cos \stackrel{\circ}{\varphi}_{j}$. Показатель экспоненты, опуская значки $j$, $-\{\ldots\}=S^{2} \cos ^{2} \varphi-2 S \bar{S}_{c} \cos \varphi+\bar{S}_{c}^{2}+S^{2} \sin ^{2} \varphi-2 S \bar{S}_{s} \sin \varphi+\bar{S}_{s}^{2}$ 
$=S^{2}+\bar{S}^{2}-2 S\left(\bar{S}_{c} \cos \varphi+\bar{S}_{s} \sin \varphi\right)$

$=S^{2}+\bar{S}^{2}-2 S \bar{S}(\cos \bar{\varphi} \cos \varphi+\sin \bar{\varphi} \sin \varphi)=S^{2}+\bar{S}^{2}-2 S \bar{S} \cos \dot{\varphi}$

$=S^{2}+\bar{S}^{2}-2 S \bar{S}+2 S \bar{S}(1-\cos \dot{\varphi})=\dot{S}^{2}+4 S \bar{S} \sin ^{2}(\dot{\varphi} / 2)$.

или $-\{\ldots\}=S^{2}+\bar{S}^{2}-2 S \bar{S} \cos \dot{\varphi}+\bar{S}^{2} \cos ^{2} \dot{\varphi}-\bar{S}^{2} \cos ^{2} \dot{\varphi}$

$=(S-\langle S\rangle)^{2}+\bar{S}^{2} \sin ^{2} \stackrel{\circ}{\varphi}$.

Пространственное распределение надшумных гдэнов

$$
\begin{aligned}
P^{\Gamma}(\mathbf{S}, \stackrel{\circ}{\varphi}) & =\prod_{j=1}^{3} P_{j}^{\Gamma}\left(S_{j}, \stackrel{\circ}{\varphi}_{j}\right), \\
P_{j}^{\Gamma}\left(S_{j}, \stackrel{\varphi}{\varphi}_{j}\right) & =\left(S_{j} / \pi\right) \exp \left\{-\stackrel{S}{S}_{j}^{2}-4 S_{j} \bar{S}_{j} \sin ^{2}\left(\stackrel{\varphi}{\varphi}_{j} / 2\right)\right\} \\
& =\left(S_{j} / \pi\right) \exp \left\{-\left(S_{j}-\left\langle S_{j}\right\rangle\right)^{2}-\bar{S}_{j}^{2} \sin ^{2} \stackrel{\circ}{\varphi}_{j}\right\},
\end{aligned}
$$

где $\mathbf{S}=\left\{S_{j}\right\}, S_{j} \geq 0, \stackrel{\circ}{\varphi}=\left\{\dot{\varphi}_{j}\right\},\left|\dot{\varphi}_{j}\right| \leq \pi$. Здесь выписаны два вида одного распределения с разными средними амплитудами $\bar{S}_{j},\left\langle S_{j}\right\rangle$ и пространственными разбросами. Ширина распределения зависит от расстояния $\stackrel{\circ}{\varphi}_{j}$ до его центра. Вблизи него $\left|\dot{\varphi}_{j}\right| \ll 1$ и распределение гауссово с шириной $1 / \bar{S}_{j}$. При удалении от центра ширина растёт. Распределение сливается с шумом при $\left\langle S_{j}\right\rangle \sim 1$, или $\cos \stackrel{\circ}{\varphi} \sim 1 / \bar{S}_{j}$. Из шума выделяется только $\left\langle S_{j}\right\rangle>1$.

Распределение надшумных гдэнов только по месту находится приближённым интегрированием по амплитуде

$$
\begin{aligned}
& P^{\Gamma}(\stackrel{\varphi}{\varphi})=\iiint_{0}^{\infty} P^{\Gamma}(\mathbf{S}, \stackrel{\varphi}{\varphi}) d^{3} \mathbf{S}=\prod_{j=1}^{3} \int_{0}^{\infty} P_{j}^{\Gamma}\left(S_{j}, \stackrel{\circ}{\varphi}_{j}\right) d S_{j}=\prod_{j=1}^{3} P_{j}^{\Gamma}\left(\dot{\varphi}_{j}\right) . \\
& P_{j}^{\Gamma}\left(\dot{\varphi}_{j}\right)=\int_{0}^{\infty} P_{j}^{\Gamma}\left(S_{j}, \dot{\varphi}_{j}\right) d S_{j}=(1 / \pi) \int_{0}^{\infty} S_{j} \exp \left\{-\left(S_{j}-\left\langle S_{j}\right\rangle\right)^{2}-\bar{S}_{j}^{2} \sin ^{2} \dot{\varphi}_{j}\right\} d S_{j} .
\end{aligned}
$$

Основной вклад в интеграл дают $S_{j} \sim\left\langle S_{j}\right\rangle$, где $\exp \{\ldots\}$ наибольшая.

$P_{j}^{\Gamma}\left(\stackrel{\varphi}{j}_{j}\right) \approx\left(\left\langle S_{j}\right\rangle / \pi\right) \exp \left\{-\bar{S}_{j}^{2} \sin ^{2} \stackrel{\circ}{\varphi}_{j}\right\} \int_{-\infty}^{\infty} \exp \left\{-\left(S_{j}-\left\langle S_{j}\right\rangle\right)^{2}\right\} d S_{j}$.

Теперь

$$
P^{\Gamma}(\stackrel{\circ}{\varphi})=\prod_{j=1}^{3} P_{j}^{\Gamma}\left(\stackrel{\varphi}{\varphi}_{j}\right), \quad P_{j}^{\Gamma}\left(\stackrel{\varphi}{j}_{j}\right)=\frac{\left\langle S_{j}\right\rangle}{\pi^{1 / 2}} \exp \left\{-\bar{S}_{j}^{2} \sin ^{2} \dot{\varphi}_{j}\right\} .
$$

При большой надшумности гдэнов $\bar{S}_{j} \gg 1$ распределение (6) узкое, т. к. $\bar{S}_{j} \sin \stackrel{\circ}{\varphi}_{j}$ быстро растёт с $\stackrel{\varphi}{\varphi}_{j}$. Можно брать $\left|\dot{\varphi}_{j}\right| \ll 1$. Тогда, опуская значки $j$,

$\stackrel{\circ}{S}^{2}+4 S \bar{S} \sin ^{2}(\stackrel{\varphi}{\varphi} / 2)=\stackrel{\leftrightarrow}{S}^{2}+S \bar{S} \dot{\varphi}^{2}$, 
$(S-\langle S\rangle)^{2}+\bar{S}^{2} \sin ^{2} \dot{\varphi}=S^{2}-2 S \bar{S} \cos \dot{\varphi}+\bar{S}^{2} \cos ^{2} \dot{\varphi}+\bar{S}^{2} \sin ^{2} \dot{\varphi}$

$=S^{2}+\bar{S}^{2}-2 S \bar{S}+2 S \bar{S}(1-\cos \dot{\varphi})=(S-\bar{S})^{2}+2 S \bar{S}(1-1+\stackrel{\varphi}{ } / 2)=\dot{S}^{2}+S \bar{S} \dot{\varphi}^{2}$.

Распределения переходят в

$$
P_{j}^{\Gamma}\left(S_{j}, \stackrel{\circ}{\varphi}_{j}\right)=\frac{S_{j}}{\pi} \exp \left\{-\stackrel{\circ}{S}_{j}^{2}-S_{j} \bar{S}_{j} \stackrel{\varphi}{\varphi}_{j}^{2}\right\}, \quad P_{j}^{\Gamma}\left(\stackrel{\varphi}{\varphi}_{j}\right)=\frac{\bar{S}_{j}}{\pi^{1 / 2}} \exp \left\{-\bar{S}_{j}^{2} \dot{\varphi}_{j}^{2}\right\},
$$

где $\left|\bar{S}_{j}\right| \gg 1$. Т. к. ширина распределения $\left\langle\dot{\varphi}_{j}^{2}\right\rangle^{1 / 2} \sim 1 / \bar{S}_{j}$, то ёмкость пространства для гдэнов $\sim \bar{S}_{j}$ растёт с их амплитудой.

Квант действия. Одномерный гдэн есть определённое колебание (2), размытое единичным шумом (3). Его амплитудная неопределённость $\left\langle S^{2}\right\rangle^{1 / 2}=1$. Вершины двух распределений различимы, если средние амплитуды отличаются более, чем на $\triangle \bar{S}=2$. Они квантуются с квантом 2. Тогда средние значения амплитуд колебаний на фоне шума совпадают с уровнями энергии квантового осциллятора $\bar{S}=2 N+1=(N+1 / 2) \hbar$, где $N$ - целое число. Т. о. постоянная Планка $\hbar$ есть удвоенная среднеквадратичная амплитуда шума двига $\hbar=2$. Однако неразличимость близких по амплитудам гдэнов лишь внешняя. Они вполне различимы по своим средним значениям.

\section{Пространство мира}

Стационарный шум флуктуаций двига, как низшее равновесное состояние, описывается гауссовым распределением плотности вероятности амплитуд гдэнов (2). В силу бесконечной делимости оно представимо многомерными колебаниями. Их одномерные части образуют измерения пространства мест (1). Пусть множество гдэнов, составляющих одномерную часть многомерных колебаний называются измеры.

Однако стационарен только весь пустой мен, а любые его части нестационарны. Нестационарность шума ведёт к отличию его распределения от гауссова и, как следствие, к изменению средних величин. Образуются отличные от нуля средние амплитуды и корреляционные связи между измерами. Если внутри каждого измера выделять противогдэн данного гдэна, то между ними также должны быть корреляции. Их набор

$$
\left\langle S_{i}^{\Gamma} S_{j}^{\Gamma}\right\rangle,\left\langle S_{j}^{\Gamma} S_{j}^{\Pi}\right\rangle,\left\langle S_{i}^{\Gamma} S_{j}^{\Gamma} S_{k}^{\Gamma}\right\rangle,\left\langle S_{i}^{\Gamma} S_{i}^{\Pi} S_{j}^{\Gamma}\right\rangle,\left\langle S_{i}^{\Gamma} S_{i}^{\Pi} S_{j}^{\Pi}\right\rangle, \ldots,
$$

где $S_{j}^{\Gamma}$ и $S_{j}^{\Pi}-$ амплитуды гдэнов и противогдэнов измера $j$. 
На перестройку распределения меняющегося шума влияет скорость релаксации его флуктуаций к новому уровню шума. Если она велика, то распределение можно считать квазистационарным гауссовым с переменными средними. Если релаксация мала, то изменение распределения отстаёт от изменения среднего уровня шума. При ослаблении шума и малой релаксации часть больших флуктуаций старого шума сохраняется и становится надшумными гдэнами, размытыми новым уровнем шума. На них переносятся корреляции (8). Амплитуды надшумных гдэнов не превышают начальный уровень шума, а их размерность определяется наличием неразрушаемых шумом корреляционных связей. Мир не создаётся, а проявляется из первошума-хаоса.

Корреляции между гдэнами разных измеров или между гдэном и противогдэном одного измера имеют одну причину и качественно похожи. Их можно взять одинаковыми. Тогда предполагается, что существование нашего пространственно однородного трёхмерного мира с симметрией между гдэнами и противогдэнами обеспечивают одинаковые связи между гдэнами разных измеров, которые могут переключаться на связи гдэна и противогдэна одного измера. Они представляются как наличие у гдэнов двух свободных связей для соединения с такими же связями гдэнов других измеров или противогдэна своего измера, где участвуют обе связи.

Случайные амплитуды $S_{j}^{\Gamma}$ и $S_{j}^{\Pi}$ измеров ј из распределения (6) записываются в виде суммы шумов. При этом парные корреляционные связи между одномерными гдэнами $\Gamma^{1}$, входящими в трёхмерный гдэн $\Gamma^{3}$ должны быть обменами амплитуд, сохраняющими их суммы.

$$
S_{j}^{\Gamma}=\bar{S}_{j}^{\Gamma}+\epsilon_{j}+g S_{j}^{\Gamma} \sum_{i=1}^{3} \eta_{j i} S_{i}^{\Gamma}, \quad \eta_{j i}=-\eta_{i j} .
$$

$\mathrm{K}$ ним добавляются уравнения для амплитуд одномерных гдэнов и противогдэнов одного измера, обеспечивающие сохранение суммы амплитуд при парных обменах и учитывающие парность связей

$$
S_{j}^{\Gamma}=\bar{S}_{j}^{\Gamma}+\epsilon_{j}+g\left(\eta_{j}+\theta_{j}\right) S_{j}^{\Gamma} S_{j}^{\Pi}, \quad S_{j}^{\Pi}=\bar{S}_{j}^{\Pi}+\epsilon_{j}-g\left(\eta_{j}+\theta_{j}\right) S_{j}^{\Gamma} S_{j}^{\Pi} .
$$

Здесь $\epsilon_{j}-$ общий для гдэнов и противогдэнов единичный шум измера $j$, $\eta_{j i}$ - единичный шум обмена амплитудами между гдэнами разных измеров, $\eta_{j}$ и $\theta_{j}$ - единичные шумы обмена амплитудами между гдэном 
и противогдэном одного измера, $g$ - коэффициенты связи между гдэнами разных измеров или между гдэном и противогдэном одного измера, которые берутся одинаковыми.

При ослабление шума первыми должны проявляются 1-местные слабо надшумные гдэны $\Gamma^{1}$, создающие 1-мерное пространство гдэнов малой ёмкости. Затем на их основе проявляются 2-местные гдэны $\Gamma^{2}=\Gamma_{1}^{1} g \Gamma_{2}^{1} g$, 1 -местные пары $\nu_{j}=\Gamma_{j}^{1} g \Pi_{j}^{1} g$ измеров $j=1,2$ и их 2 -местные суммы $\nu_{1} \nu_{2}$. (Здесь и далее последняя $g$-связь соединяет последний и первый гдэны.) Они образуют 2-мерное пространство гдэнов и два 1-мерных пространства пар $\nu_{j}$. Дальнейшее ослабление шума вызывает проявление 1-местных пар $\nu_{j}=\Gamma_{j}^{1} g \Pi_{j}^{1} g$ измеров $j=1,2,3,2$-местных $\nu_{j} \nu_{k}$,

$\nu_{j k}=\Gamma_{j}^{1} g \Pi_{j}^{1} g \Gamma_{k}^{1} g \Pi_{k}^{1} g, 3$-местных $\nu_{1} \nu_{2} \nu_{3}, \nu_{123}=\Gamma_{1}^{1} g \Pi_{1}^{1} g \Gamma_{2}^{1} g \Pi_{2}^{1} \Gamma_{3}^{1} g \Pi_{3}^{1} g$ и более длинных сочетаний. Этот процесс может продолжаться и дальше, образуя миры бо́льших размерностей.

Пространство нашего мира имеет три измерения. Оно однородно, изотропно и замкнуто на себе - места $\varphi_{j}= \pm \pi$ совпадают. Относительно любого места его можно представить, как состоящее из собственно пространства мира и зеркального ему пространства противомира, в котором гдэны и противогдэны взаимно заменены. Мир только кажется бесконечным при наблюдении из его малой части за малое время.

\section{Взаимодействие гдэнов}

Корреляционная $g$-связь $(9,10)$, объединяя одноместные гдэны в трёхместные создаёт основу нашего трёхмерного пространства. Однако этого недостаточно для существования сложного и развивающегося мира. Требуется взаимодействие между трёхместными гдэнами, позволяющее им образовывать всё более крупные строения. Эту роль берёт на себя взаимодействие гдэнов через двиг. Гдэн есть порождение двига, который поддерживает его существование и определяет его свойства. Но и гдэн, будучи частью двига, влияет на него. Тогда другой гдэн в возмущённом двиге также будет меняться.

Гдэн, как целое, определяется своими частотой $\Omega$ и амплитудой $\mathrm{S}, \mathrm{a}$ его место $\varphi$ выражает отношение к другим гдэнам (1). Двиг влияет на гдэн через частоту и амплитуду. Их изменение описывается производными $\dot{\Omega}$ и $\dot{S}$. При достаточно слабом влиянии двига можно считать, что гдэн (т. е. $S$ и $\Omega$ ) сохраняется, а изменение перенести на его перемещение 
$\dot{\Omega}=\ddot{\varphi}$. Тогда в возмущённом двиге гдэн ускоряется.

При заданной частоте гдэна $\Omega=1$, его усреднённое влияние на двиг определяется средней амплитудой $\bar{S}$ и выражается функцией $U(\bar{S})$. Если $\bar{S}$ постоянно, то двиг постоянен, и другой гдэн в нём не меняется. Ускоряет гдэн переменное влияние двига. Для воздействующего гдэна с постоянным во времени распределением, это сводится к влиянию градиента $\mathrm{U}: \ddot{\varphi}=-\partial_{\varphi} U$, или $U+(\dot{\varphi})^{2} / 2$ постоянно. Т. о. функция $U(\bar{S})$ есть потенциал воздействия гдэна на другой гдэн через двиг.

Если наш мир образовался из начального шума при его ослаблении с сохранением флуктуаций, то он состоит из основы с амплитудой $S_{0}$ и возвышающимися над ней различимыми между собой частями гдэнов с амплитудами $S_{u}: S=S_{0}+S_{u}, \bar{S}=S_{0}+\bar{S}_{u}$ (см. "Среда мира"). При $S_{0} \gg \bar{S}_{u}, U(\bar{S})$ разлагается в ряд $U(\bar{S})=U\left(S_{0}\right)+U_{S} \bar{S}_{u}+U_{S S} \bar{S}_{u}^{2}+\ldots$, где $U_{S}=d_{S} U\left(S=S_{0}\right), U_{S S}=d_{S S}^{2} U\left(S=S_{0}\right)$ - производные. Выбор $U\left(S_{0}\right)$ за начало отсчёта $U\left(S_{0}\right)=0$ даёт

$$
U(\bar{S})=U\left(\bar{S}_{u}\right)=U_{S} \bar{S}_{u}+U_{S S} \bar{S}_{u}^{2} / 2=U_{\gamma}\left(\bar{S}_{u}\right)+U_{G}\left(\bar{S}_{u}\right), U_{\gamma} \gg U_{G},
$$

где малость $U_{G} / U_{\gamma}$ связана с малостью $\bar{S}_{u} / S_{0}$.

После введения противогдэнов и соответствующего уменьшения пространства мест вдвое до $\mid \stackrel{\varphi}{\mid} \leq \pi / 2$, амплитуда $\bar{S}_{u}$ становится для противогдэнов отрицательной. Теперь потенциал состоит из нечётной $U_{\gamma}=U_{S} \bar{S}_{u}$ и чётной $U_{G}=U_{S S} \bar{S}_{u}^{2} / 2$ по амплитудам частей. Его зависимость от места $\stackrel{\odot}{\varphi}$ находится из распределения гдэна $(7)$, которое меняется на $P_{j}^{\Gamma}\left(S_{j}, \dot{\varphi}_{j}\right)=P_{j}^{\Gamma}\left(S_{u j}, \dot{\varphi}_{j}\right)=\left(S_{0} / \pi\right) \exp \left\{-\dot{S}_{u j}^{2}-\dot{\phi}_{j}^{2}\right\}$, где $\dot{\phi}_{j}=S_{0} \stackrel{\circ}{j}_{j}$. После замены $\stackrel{\circ}{j}_{j}$ на $\stackrel{\circ}{\phi}_{j}: P_{j}^{\Gamma}\left(\stackrel{\circ}{S}_{u j}, \dot{\phi}_{j}\right)=P_{j}^{\Gamma}\left(S_{u j}, \stackrel{\varphi}{\varphi}_{j}\right) / S_{0}$ или

$$
P^{\Gamma}\left(\stackrel{\mathbf{S}}{u}_{u}, \stackrel{\circ}{\phi}\right)=\prod_{j=1}^{3} P_{j}^{\Gamma}\left(\stackrel{S}{S}_{u j}, \stackrel{\phi}{\phi}_{j}\right), \quad P_{j}^{\Gamma}\left(S_{u j}, \stackrel{\phi}{\phi}_{j}\right)=\frac{1}{\pi} \exp \left\{-\stackrel{S}{S}_{u j}^{2}-\stackrel{\circ}{\phi}_{j}^{2}\right\},
$$

где $\stackrel{\circ}{\mathbf{S}}_{u}=\left\{S_{u j}-\overline{S_{u j}}\right\}, \stackrel{\circ}{\phi}=\left\{\phi_{j}-\bar{\phi}_{j}\right\}, \bar{\phi}_{j}=S_{0} \bar{\varphi}_{j}$.

Пространственная часть распределения записывается через амплитуду $S_{0}$ основы, а амплитудная часть - через возвышение $S_{u}$ над ней.

Средние амплитуды гдэнов $\Gamma_{j}^{1}$ берутся одинаковыми $\bar{S}_{u j}=\bar{S}_{u}$, а $P^{\Gamma}(\stackrel{\mathrm{S}}{\mathbf{S}}, \stackrel{\circ}{\phi})$ преобразуется к распределению $P^{\Gamma}\left(S_{u}, \stackrel{\circ}{\phi}\right)$ амплитуды $S_{u} \equiv S_{u 1}+S_{u 2}+S_{u 3}$ гдэна $\Gamma^{3}$. 


$$
\begin{gathered}
P^{\Gamma}\left(S_{u}, \stackrel{\circ}{\mathbf{\phi}}\right)=\iint P_{1}^{\Gamma}\left(\stackrel{\circ}{S}_{u 1}, \stackrel{\circ}{\phi}_{1}\right) P_{2}^{\Gamma}\left(\stackrel{\leftrightarrow}{S}_{u 2}, \stackrel{\circ}{\phi}_{2}\right) P_{3}^{\Gamma}\left(\stackrel{\leftrightarrow}{S}_{u}-S_{u 1}-S_{u 2}, \stackrel{\phi}{\phi}_{3}\right) d S_{u 1} d S_{u 2} \\
=\pi^{-3} \iint \exp \left\{-\stackrel{\circ}{S}_{u 1}^{2}-\stackrel{\circ}{S}_{u 2}^{2}-\left(S_{u}-S_{u 1}-S_{u 2}-\bar{S}_{u}\right)^{2}-\stackrel{\circ}{\phi}^{2}\right\} d S_{u 1} d S_{u 2},
\end{gathered}
$$

где $\dot{\phi}^{2}=\grave{\phi}_{1}^{2}+\grave{\phi}_{2}^{2}+\stackrel{\phi}{2}_{3}^{2}$. Основной вклад в интеграл дают $S_{u 1} \sim \bar{S}_{u}$ и $S_{u 2} \sim \bar{S}_{u}$, где экспонента наибольшая, а её изменение наименьшее. Тогда $P^{\Gamma}\left(S_{u}, \stackrel{\phi}{\phi}\right) \approx \pi^{-2} \exp \left\{-\left(S_{u}-S_{\Gamma}\right)^{2}-\dot{\phi}^{2}\right\}$,

где $S_{\Gamma}=3 \bar{S}_{u}-$ средняя амплитуда трёхместного гдена, $\dot{\phi}-$ расстояние от его центра. Пространственное распределение

$$
P^{\Gamma}(\stackrel{\circ}{\phi})=\int_{-\infty}^{\infty} P^{\Gamma}\left(S_{u}, \stackrel{\circ}{\phi}\right) d S_{u}=\pi^{-2} e^{-\dot{\phi}^{2}} \int_{-\infty}^{\infty} e^{-\left(S_{u}-S_{\Gamma}\right)^{2}} d S_{u}
$$

Теперь распределение трёхместного гдэна

$$
P^{\Gamma}\left(S_{u}, \stackrel{\circ}{\boldsymbol{\phi}}\right)=\pi^{-2} \exp \left\{-\left(S_{u}-S_{\Gamma}\right)^{2}-\stackrel{\circ}{\phi}^{2}\right\}, \quad P^{\Gamma}(\stackrel{\circ}{\boldsymbol{\phi}})=\pi^{-3 / 2} e^{-\stackrel{\circ}{\phi}^{2}}
$$

Пространственное распределение потенциала воздействия через двиг определяется пространственной зависимостью средней амплитуды гдэна, т. е. его условным средним для распределения (12)

$$
U(\stackrel{\circ}{\phi})=\left\langle U\left(S_{u}\right) \mid \stackrel{\circ}{\phi}\right\rangle=\int_{0}^{\infty} U\left(S_{u}\right) P^{\Gamma}\left(S_{u}, \stackrel{\circ}{\phi}\right) d S_{u} .
$$

Т. к. основной вклад в интеграл дают $S_{u} \sim S_{\Gamma}$, то

$$
U(\stackrel{\circ}{\phi}) \approx U\left(S_{\Gamma}\right) \pi^{-2} e^{-\stackrel{\circ}{\phi}^{2}} \int_{-\infty}^{\infty} e^{-\left(S_{u}-S_{\Gamma}\right)^{2}} d S_{u}=U\left(S_{\Gamma}\right) \pi^{-3 / 2} e^{-\stackrel{\circ}{\phi}^{2}}
$$

Подставив разложение (11) получим потенциал воздействия гдэнов через двиг

$$
U(\stackrel{\circ}{\boldsymbol{\phi}})=U_{\gamma}(\stackrel{\circ}{\boldsymbol{\phi}})+U_{G}(\stackrel{\circ}{\boldsymbol{\phi}}), U_{\gamma}(\stackrel{\circ}{\boldsymbol{\phi}})=\gamma e^{-\stackrel{\circ}{ }^{2}}, U_{G}(\stackrel{\circ}{\boldsymbol{\phi}})=G e^{-\stackrel{\circ}{ }^{2}}, \stackrel{\circ}{\phi}=S_{0} \stackrel{\circ}{\varphi},
$$

где $\gamma=\gamma_{u} S_{\Gamma}, \quad G=G_{u} S_{\Gamma}^{2}, \quad \gamma_{u}=\pi^{-3 / 2} U_{S}, \quad G_{u}=\pi^{-3 / 2} U_{S S} / 2$,

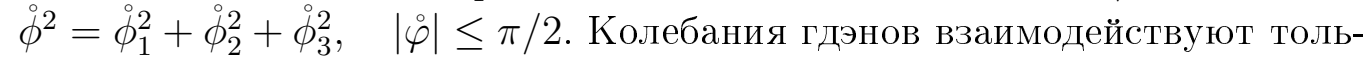
ко местно, при совпадение их фаз. Но сам гдэн состоит из определённых колебаний (1), размытых шумом (3) до распределения (12). Пространственное распределение потенциала (13) связано с этим размытием, т. 
е. определяется шумом. Следовательно гдэны взаимодействуют посредством шума. Действует же потенциал не на шум, а на определённое колебание гдэна (центр распределения), и ускоряет его.

Знаки постоянных воздействия $\gamma_{u}, G_{u}$ определяют его качество. Если $\gamma_{u}>0$, то гдэн отталкивается от гдэна и притягивается противогдэном. Если $G_{u}>0$, то любые гдэны и противогдэны отталкиваются. Такие знаки соответствуют нашему устойчивому и развивающемуся миру.

Т. к. $\gamma$-потенциал $U_{\gamma}$ является наибольшим (11), то исследование взаимодействия гдэнов начнём с него. Рассмотрим одномерное $\gamma$-взаимодействие трёхместных гдэнов $\Gamma^{3}$ с равными по модулю амплитудами $S_{\Gamma}$. В описании взаимодействия используются следущие обозначения

$$
U(r)=e^{-r^{2}}, \quad f(r)=r U, \quad f_{r}(r)=\left(1-2 r^{2}\right) U, \quad f_{r r}(r)=\left(4 r^{3}-6 r\right) U .
$$

Взаимодействие двух гдэнов. Для описания взаимодействия двух трёхместных гдэнов $\Gamma_{1}$ и $\Gamma_{2}$ выбрана система отсчёта, в которой движение симметрично. Места гдэнов $\phi_{1}$ и $\phi_{2}, \phi=\phi_{1}-\phi_{2}=2 \phi_{1}$. Потенциал взаимодействия (13), записанный в единицах $\gamma$, есть $U(\phi)= \pm e^{-\phi^{2}}$.

Верхний (нижний) знак отмечает взаимодействие гдэн-гдэн

(гдэн-противогдэн). Действующие на гдэны удсилы - удельные (на единицу амплитуды) силы $f_{1}=-f_{2}=-d_{\phi} U= \pm 2 \phi U(\phi)$. Уравнения относительного движения (урдвиж) $\ddot{\phi}=2 f_{1}= \pm 4 \phi U(\phi)$.

Т. к. $\phi=\phi(t)$, то $t=t(\phi)$ и $\ddot{\phi}=d_{\phi}(\dot{\phi})^{2} / 2=-2 d_{\phi} U(\phi)$. Тогда $(\dot{\phi})^{2} / 2+2 U(\phi)=E,(\dot{\phi})^{2}=2(E-2 U)$,

где $\mathrm{E}$ - удельная полная энергия относительного движения гдэнов. Точки поворота $\phi_{m}$ находятся из $\dot{\phi}\left(\phi_{m}\right)=0: E= \pm 2 e^{-\phi_{m}^{2}}, \phi_{m}^{2}=\ln ( \pm 2 / E)$.

Отталкивание гдэн-гдэн. Если $0<E<2$, то при встречном движении имеется отражение в точке поворота. Два одинаковых гдэна не могут быть в одном месте (состоянии). Это свойство фермионов. Оно обусловлено $\gamma$-отталкиванием при достаточно малой энергии Е. Следовательно гдэны - фермионы.

Притяжение гдэн-противогдэн (маятник ГП). Если $-2<E<0$, то в потенциале $U(\phi)$ существует нелинейный мягкий маятник с амплитудой $\phi_{m}$. Вблизи дна потенциала $\phi^{2} \ll 1$ при $E \gtrsim-2$ маятник линеен $\ddot{\phi}=-4 \phi$ с частотой $\omega_{\gamma}=2$ или

$$
\omega_{\gamma}=2 \gamma^{1 / 2}
$$

Маятник ГПГ 
Два гдэна Г1, Г2 и противогдэн П с равными по модулю амплитудами имеют места $\phi_{1}, \phi_{2}$ и $\phi_{0}$. Разности мест

$\phi_{12}=\phi_{1}-\phi_{2}, \phi_{10}=\phi_{1}-\phi_{0}, \phi_{20}=\phi_{2}-\phi_{0}$.

Потенциалы воздействия на них (13), в единицах $\gamma$

$U_{1}=e^{-\phi_{12}^{2}}-e^{-\phi_{10}^{2}}, \quad U_{2}=e^{-\phi_{12}^{2}}-e^{-\phi_{20}^{2}}, \quad U_{0}=-e^{-\phi_{10}^{2}}-e^{-\phi_{20}^{2}}$.

Сумма потенциалов $U=U_{1}+U_{2}+U_{0}=2\left(e^{-\phi_{12}^{2}}-e^{-\phi_{10}^{2}}-e^{-\phi_{20}^{2}}\right)$.

Удсилы $f_{j}=-\partial_{\phi_{j}} U_{j}=-\partial_{\phi_{j}} U / 2, j=1,2,0: \quad f_{1}=2 \phi_{12} e^{-\phi_{12}^{2}}-2 \phi_{10} e^{-\phi_{10}^{2}}$, $f_{2}=-2 \phi_{12} e^{-\phi_{12}^{2}}-2 \phi_{20} e^{-\phi_{20}^{2}}, \quad f_{0}=2 \phi_{10} e^{-\phi_{10}^{2}}+2 \phi_{20} e^{-\phi_{20}^{2}}$.

Урдвиж с учётом $\phi_{12}=\phi_{10}-\phi_{20}$ есть

$\ddot{\phi}_{10}=2 \phi_{12} e^{-\phi_{12}^{2}}-4 \phi_{10} e^{-\phi_{10}^{2}}-2 \phi_{20} e^{-\phi_{20}^{2}}$,

$\ddot{\phi}_{20}=-2 \phi_{12} e^{-\phi_{12}^{2}}-2 \phi_{10} e^{-\phi_{10}^{2}}-4 \phi_{20} e^{-\phi_{20}^{2}}, \quad \ddot{\phi}_{0}=2 \phi_{10} e^{-\phi_{10}^{2}}+2 \phi_{20} e^{-\phi_{20}^{2}}$.

Если $\phi_{2}<\phi_{0}<\phi_{1}$ (П между Г1 и Г2), то в полном потенциале U выполняется $\phi_{12}>\phi_{10}, \phi_{20}$ и $e^{-\phi_{12}^{2}}<e^{-\phi_{10}^{2}}, e^{-\phi_{20}^{2}}$. Возможно $\mathrm{U}<0$ и связанное состояние (маятник ГПГ) при достаточно малой полной энергии. В нём выделяются три части движения гдэнов:

При $\left|\phi_{10}\right| \ll 1$, П движется вблизи $\Gamma_{1}$. Т. к. здесь нелинейные члены малы, то $\ddot{\phi}_{10} \approx-4 \phi_{10}, \ddot{\phi}_{0} \approx 2 \phi_{10}, \ddot{\phi}_{20} \approx-2 \phi_{10}, \phi_{2} \approx 0$. П проходит вблизи $\Gamma_{1}$ в состоянии линейного маятника $\Gamma_{1} \Pi$ с частотой $\omega_{\gamma}=2$, а $Г 2$ движется по инерции $f_{2} \approx 0$.

При $\left|\phi_{20}\right| \ll 1,-$ линейный маятник $\Gamma_{2} \Pi$, а Г2 движется по инерции.

При $\phi_{20} \approx-\phi_{10}$, П движется по инерции на энергетическом перевале между $\Gamma_{1}$ и $\Gamma_{2}$.

Т. о. движение маятника ГПГ состоит из последовательных прохождений маятников $\Gamma_{1} \Pi, \Gamma_{2} \Pi$ и перевала потенциала между ними. Его период оценивается временем прохождения двух точек поворота и перевала, что больше периода линейного маятника ГП.

Взаимодействие двух маятников ГП

Пусть имеется две пары гдэн-противогдэн $\Gamma_{1} \Pi_{1}$ и $\Gamma_{2} \Pi_{2}$ с равными по модулю амплитудами гдэнов. В рассматриваемом для них одномерном движении их места $\phi_{1}^{\Gamma}, \phi_{1}^{\Pi}, \phi_{2}^{\Gamma}, \phi_{2}^{\Pi}$. Вводятся разности мест в парах $\xi_{1}=\phi_{1}^{\Gamma}-\phi_{1}^{\Pi}, \xi_{2}=\phi_{2}^{\Gamma}-\phi_{2}^{\Pi}$ и расстояние $r$ между центрами пар.

Действующие на гдэны удсилы в единицах $2 \gamma$ (13) записываются в соответствие с обозначениями (14)

$f_{1}^{\Gamma}=-f\left(\phi_{1}^{\Gamma}-\phi_{1}^{\Pi}\right)+f\left(\phi_{1}^{\Gamma}-\phi_{2}^{\Gamma}\right)-f\left(\phi_{1}^{\Gamma}-\phi_{2}^{\Pi}\right)$,

$f_{1}^{\Pi}=f\left(\phi_{1}^{\Gamma}-\phi_{1}^{\Pi}\right)-f\left(\phi_{1}^{\Pi}-\phi_{2}^{\Gamma}\right)+f\left(\phi_{1}^{\Pi}-\phi_{2}^{\Pi}\right)$,

$f_{2}^{\Gamma}=-f\left(\phi_{1}^{\Gamma}-\phi_{2}^{\Gamma}\right)+f\left(\phi_{1}^{\Pi}-\phi_{2}^{\Gamma}\right)-f\left(\phi_{2}^{\Gamma}-\phi_{2}^{\Pi}\right)$,

$f_{2}^{\Pi}=f\left(\phi_{1}^{\Gamma}-\phi_{2}^{\Pi}\right)-f\left(\phi_{1}^{\Pi}-\phi_{2}^{\Pi}\right)+f\left(\phi_{2}^{\Gamma}-\phi_{2}^{\Pi}\right)$. 
Урдвиж в единицах $2 \gamma=\omega_{\gamma}^{2} / 2(15)$

$\ddot{\xi}_{1}=f_{1}^{\Gamma}-f_{1}^{\Pi}=-f\left(\xi_{1}\right)+f\left(r+\left(\xi_{1}-\xi_{2}\right) / 2\right)-f\left(r+\left(\xi_{1}+\xi_{2}\right) / 2\right)-f\left(\xi_{1}\right)$

$+f\left(r-\left(\xi_{1}+\xi_{2}\right) / 2\right)-f\left(r-\left(\xi_{1}-\xi_{2}\right) / 2\right)$,

$\ddot{\xi}_{2}=f_{2}^{\Gamma}-f_{2}^{\Pi}=-f\left(r+\left(\xi_{1}-\xi_{2}\right) / 2\right)+f\left(r-\left(\xi_{1}+\xi_{2}\right) / 2\right)-f\left(\xi_{2}\right)$

$-f\left(r+\left(\xi_{1}+\xi_{2}\right) / 2\right)+f\left(r-\left(\xi_{1}-\xi_{2}\right) / 2\right)-f\left(\xi_{2}\right)$,

$2 \ddot{r}=f_{1}^{\Gamma}+f_{1}^{\Pi}-f_{2}^{\Gamma}-f_{2}^{\Pi}=-f\left(\xi_{1}\right)+f\left(r+\left(\xi_{1}-\xi_{2}\right) / 2\right)$

$-f\left(r+\left(\xi_{1}+\xi_{2}\right) / 2\right)+f\left(\xi_{1}\right)-f\left(r-\left(\xi_{1}+\xi_{2}\right) / 2\right)+f\left(r-\left(\xi_{1}-\xi_{2}\right) / 2\right)$

$+f\left(r+\left(\xi_{1}-\xi_{2}\right) / 2\right)-f\left(r-\left(\xi_{1}+\xi_{2}\right) / 2\right)+f\left(\xi_{2}\right)-f\left(r+\left(\xi_{1}+\xi_{2}\right) / 2\right)$

$+f\left(r-\left(\xi_{1}-\xi_{2}\right) / 2\right)-f\left(\xi_{2}\right)=$

$=2\left[f\left(r+\left(\xi_{1}-\xi_{2}\right) / 2\right)-f\left(r+\left(\xi_{1}+\xi_{2}\right) / 2\right)-f\left(r-\left(\xi_{1}+\xi_{2}\right) / 2\right)+f\left(r-\left(\xi_{1}-\xi_{2}\right) / 2\right)\right]$.

При $|\xi| \ll r$ удсилы разлагаются в ряд по степеням $\xi$ до $\xi^{3}$. Используя обозначения $f=f(r), f_{r}=f_{r}(r), f_{r r}=f_{r r}(r), f_{r r r}=f_{r r r}(r)$ из (14), урдвиж записываются как

$\ddot{\xi}_{1}=-2\left(\xi_{1}-\xi_{1}^{3}\right)+f_{r}\left(\xi_{1}-\xi_{2}\right)+f_{r r r}\left(\xi_{1}-\xi_{2}\right)^{3} / 24-f_{r}\left(\xi_{1}+\xi_{2}\right)-f_{r r r}\left(\xi_{1}+\xi_{2}\right)^{3} / 24$ $=-2\left(\xi_{1}-\xi_{1}^{3}\right)-2 f_{r} \xi_{2}-f_{r r r}\left(\xi_{1}^{3}+3 \xi_{1}^{2} \xi_{2}+3 \xi_{1} \xi_{2}^{2}+\xi_{2}^{3}-\xi_{1}^{3}+3 \xi_{1}^{2} \xi_{2}-3 \xi_{1} \xi_{2}^{2}+\xi_{2}^{3}\right) / 24$

$=-2\left(\xi_{1}-\xi_{1}^{3}\right)-2 f_{r} \xi_{2}-f_{r r r}\left(3 \xi_{1}^{2} \xi_{2}+\xi_{2}^{3}\right) / 12$,

$\ddot{\xi}_{2}=-2\left(\xi_{2}-\xi_{2}^{3}\right)-2 f_{r} \xi_{1}-f_{r r r}\left(3 \xi_{2}^{2} \xi_{1}+\xi_{1}^{3}\right) / 12$,

$\ddot{r}=2 f+f_{r r}\left(\xi_{1}-\xi_{2}\right)^{2} / 4-2 f-f_{r r}\left(\xi_{1}+\xi_{2}\right)^{2} / 4=-f_{r r} \xi_{1} \xi_{2}$.

Тогда

$\ddot{\xi}_{1}=-2\left(\xi_{1}-\xi_{1}^{3}\right)-2 f_{r} \xi_{2}-f_{r r r}\left(3 \xi_{1}^{2} \xi_{2}+\xi_{2}^{3}\right) / 12$,

$\ddot{\xi}_{2}=-2\left(\xi_{2}-\xi_{2}^{3}\right)-2 f_{r} \xi_{1}-f_{r r r}\left(3 \xi_{2}^{2} \xi_{1}+\xi_{1}^{3}\right) / 12, \quad \ddot{r}=-f_{r r} \xi_{1} \xi_{2}$.

T. к. $r$ меняется во втором приближении, то его изменением в уравнениях для $\xi_{1}$ и $\xi_{2}$ пренебрегалось с самого начала.

Первое приближение (линейное)

В линейном приближении урдвиж

$\ddot{\xi}_{1}=-2 \xi_{1}-2 f_{r} \xi_{2}, \quad \ddot{\xi}_{2}=-2 \xi_{2}-2 f_{r} \xi_{1}, \quad \ddot{r}=0$.

Решение ищется в виде $\xi_{j}=a_{j} e^{-i \omega t}$.

$-\omega^{2} a_{1}=-2 a_{1}-2 f_{r} a_{2}, \quad-\omega^{2} a_{2}=-2 a_{2}-2 f_{r} a_{1}$.

Собственные частоты в единицах $\omega_{\gamma}^{2} / 2$ есть $\omega_{ \pm}^{2}=2\left(1 \mp f_{r}\right)$, или

$\omega_{ \pm}^{2}=\omega_{\gamma}^{2}\left(1 \mp f_{r}\right) \cdot f_{r}$ описывает влияние соседней пары: $f_{r}<0$ при $r^{2}>1 / 2$. Собственные частоты $\omega_{ \pm}$соответствуют собственным колебаниям в переменных $\xi_{ \pm}=\left(\xi_{1} \pm \xi_{2}\right)$ :

$\ddot{\xi}_{ \pm}=-2 \xi_{1}-2 f_{r} \xi_{2} \mp\left(2 \xi_{2}+2 f_{r} \xi_{1}\right)=-2\left(1 \pm f_{r}\right)\left(\xi_{1} \pm \xi_{2}\right)$.

$\xi_{+}$колеблются с меньшей частотой $\omega_{-}$, а $\xi_{-}-$с большей $\omega_{+}$.

Если пары колеблются в фазе $\xi_{1} \approx \xi_{2}$ и $r^{2}>1 / 2$, то удсила менышеет с расстоянием. Пара притягивает ближний к ней гдэн другой пары и отталкивает дальний, но слабее. Центры пар сближаются - пары при- 
тягиваются.

Если пары колеблются в противофазе $\xi_{1} \approx-\xi_{2}$, то пары отталкиваются.

Второе приближение (нелинейное)

В нелинейном приближении находится изменение расстояния между парами ГП

$$
\ddot{r}=-f_{r r} \xi_{1} \xi_{2}=-f_{r r}\left(\xi_{+}^{2}-\xi_{-}^{2}\right) / 4,
$$

где справа стоят решения первого приближения. Подставляя собственные гармоники в виде $\xi_{ \pm}=a_{ \pm} \cos \Phi_{ \pm}, \Phi_{ \pm}=\omega_{ \pm} t+\phi_{ \pm}$, получим $\xi_{+}^{2}-\xi_{-}^{2}=a_{+}^{2} \cos ^{2} \Phi_{+}-a_{-}^{2} \cos ^{2} \Phi_{-}=a_{+}^{2}\left(1+\cos 2 \Phi_{+}\right) / 2-a_{-}^{2}\left(1+\cos 2 \Phi_{-}\right) / 2$ $=\left(a_{+}^{2}-a_{-}^{2}+a_{+}^{2} \cos 2 \Phi_{+}-a_{-}^{2} \cos 2 \Phi_{-}\right) / 2$.

Удсила взаимодействия пар $f=\ddot{r}=-f_{r r}\left(a_{+}^{2}-a_{-}^{2}+a_{+}^{2} \cos 2 \Phi_{+}-a_{-}^{2} \cos 2 \Phi_{-}\right) / 8$. Без учёта вторых гармоник (усреднение за период колебаний) удсила медленного взаимодействия пар ГП есть

$$
\bar{f}=-f_{r r}\left(a_{+}^{2}-a_{-}^{2}\right) / 8
$$

Из (14) $f_{r r}=2 r\left(2 r^{2}-3\right) U$ меняет знак при $r^{2}=r_{0}^{2}=3 / 2$.

Если пары ГП колеблются противофазно $a_{-}^{2}>a_{+}^{2}$, то $\bar{f}>0$ при $r>r_{0}$ (отталкивание пар) и $\bar{f}<0$ при $r<r_{0}$ (притяжение пар). $r_{0}-$ точка неустойчивого равновесия. Если пары ГП колеблются софазно $a_{-}^{2}<a_{+}^{2}$, то $r_{0}$ - точка устойчивого равновесия.

\section{Среда мира}

Результаты, полученные по взаимодействию через двиг, позволяют продолжить исследование развития и строения среды нашего мира. Было показано, что мир может возникнуть из начального внепространственного шума $(2,3)$ при его ослаблении с сохранением флуктуаций. Сохранённые флуктуации выделяются из шума, когда их амплитуды более чем вдвое превышают среднюю амплитуду шума. Возможно, что каждое уменьшение шума в $\sim 2$ раза сопровождается образованием новой ступени надшумных гдэнов из сохранённых флуктуаций предыдущей. Здесь, в отличие от внепространственного первошума, флуктуации шума имеют место и ширину $\sim 1 / S$, где $S \sim 2^{n}$ - их амплитуда, суммарная по всем ступеням надшумности, $n-$ число ступеней.

Пространственная неопределённость гдэнов $\Delta \phi=S \Delta \varphi \sim 2$. Гдэны внешне не различаются, если расстояние между их центрами $r<2$. В 
этом случае вместо частокола гдэнов, ступень состоит из их плато, над которым флуктуирует текущий шум. Пространственное постоянство ступени ведёт к её незаметности, а энергия может отсчитываться от энергетического уровня ступени. Заметными остаются только возвышения над ней. Это текущий шум с дисперсией, взятой за единицу действия, и надшумные гдэны, образующие строение мира.

Можно сделать простую оценку числа ступеней ослабления шума. Сейчас радиус вселенной оценивается в $10^{27}$ метра. Размер гдэна не более радиуса электрона, который меньше $10^{-22}$ м [11] (см. "Частицы"). Если вселенная занимает всё пространство мест, то вдоль каждой прямой в ней может поместиться не менее $10^{49}$ гдэнов, или $\gtrsim 10^{147}$ гдэнов во вселенной. Для этого необходима амплитуда гдэна $\gtrsim 10^{49} \sim 2^{163}$ в единицах шума, что даёт число ступеней надшумности $n \gtrsim 163$ при удвоении амплитуд от ступени к ступени. Если сейчас шум $\sim \hbar=10^{-27}$ эргс, то первошум имеет амплитуду $S_{0} \gtrsim 10^{22}$ эргс. Такая мощь скрывается за "пустотой" нашего пространства.

Наш мир расположен на мощной, но незаметной для нас основе. Флуктуации его шума дают нулевые колебания "физического вакуума", над которыми выделяются относительно редкие частицы. Эти флуктуации пространственно различимы, в отличие от первошума. Хотя частицы заметно размыты шумом (квантовая неопределённость), они имеют пространственное расположение.

Гдэны и противогдэны $\gamma$-взаимодействием соединяются в ГП-пары $\Gamma^{3} \gamma \Pi^{3}$, которые при определённых условиях связываются в устойчивую ГП-среду. Относительный сдвиг гдэна и противогдэна в паре определяет поляризацию этой среды.

Кроме ГП-пар существует множество одноместных пар $\nu_{j}=\Gamma_{j} g g \Pi_{j}$. Это нейтрино (см. "Частицы"). Пусть составленная из них среда называется нейтр $N_{j}$ измера $\mathrm{j}$. Трёхмерный нейтр представлен их суммой $N=N_{1}+N_{2}+N_{3}$.

Составляющие нейтрино гдэны и противогдэны, колеблясь противофазно, в свободном состоянии имеют равные по величине и противоположные по знаку амплитуды. Нейтрино, в среднем, нейтральны и участвуют только во всеобщем G-взаимодействие (13), которое намного слабее $\gamma$-взаимодействия. В поляризованной ГП-среде его можно не учитывать, но в отсутствии поляризации оно может быть основным.

Т. о. материя нашего мира составлена, в основном, из ГП-пар и нейтрино. Её плотность $\rho$ не менее плотности ГП-среды. Она грубо оце- 
нивается через массу ГП-пары (порядка массы нейтрино $10^{-37}$ кг - см. "Частицы") и расстояние $r$ между ними (не более размера электрона $\left.\sim 10^{-22} \mathrm{м}\right)$. Тогда $\rho \gtrsim 10^{29} \mathrm{kг} / \mathrm{M}^{3}$, что много больше плотности ядер атомов. Плотность вещества во вселенной оценивается в $\sim 10^{-26} \mathrm{kг} / \mathrm{m}^{3}$. Разница на 55 порядков или более. Но ГП-среда только возвышается над верхней ступенью основания, которая сама более чем на 49 порядков больше современного шума $\sim \hbar$. Тогда вся известная науке энергия-масса ничтожно мала по сравнению с энергией её основы - разница превышает 100 порядков.

\section{Поляризация}

Гдэны и противогдэны $\gamma$-взаимодействием соединяются в пары $\Gamma^{3} \gamma \Pi^{3}$, которые при определённых (пока неизвестных) условиях образуют устойчивую среду. В ней $\gamma$-взаимодействие смещает гдэны пар и меняет расстояние между парами. Относительный сдвиг гдэна и противогдэна в паре определяет поляризацию этой среды.

Рассмотрим однородную ГП-среду, занимающую всё пространство мест гдэнов. Пусть она состоит из ГП-пар, содержащих гдэны с одинаковыми средними амплитудами. В невозмущённом равновесном состоянии гдэн и противогдэн в паре совмещены, а в возмущённом - разделены. За координатные оси трёхмерного пространства возьмём измеры $(9,10)$, а полученное объединение связанных маятников ГП используем для описания ГП-среды. Будут использованы три модели расположения ГП-пар: две решётки куба (простая, объёмноцентрированная) и непрерывное распределение.

Каждый гдэн имет определённое место только в среднем. Его распределение $(6,7)$ размыто по месту с дисперсией $\left\langle\phi^{2}\right\rangle=1$. Даже когда гдэн и противогдэн совмещены, разность их мест шумит с удвоенной дисперсией $\left\langle\left(\phi^{\Gamma}-\phi^{\Pi}\right)^{2}\right\rangle=2$. На этом фоне малое разделение $\Gamma^{3}$ и $\Pi^{3}$ внешне не выделяется, но оно есть как средняя величина. А большие и быстрые изменения шума при усреднение за относительно медленные времена перемещений гдэнов взаимно уничтожаются и становятся незаметными, не мешая составлять для средних вполне определённые соотношения.

Точное рассмотрения динамики возмущений в такой среде невозможно. Нужны упрощённые приближённые подходы, одним из которых является учёт взаимодействия только с ближайшими соседями и разложе- 
ние по степени возмущения. Получение качественных результатов возможно уже в линейном приближении с добавлением нужных нелинейных дополнений.

\section{Простая решётка куба.}

В простой решётке куба ГП-пары занимают его вершины. Выделяются ось $l$ (продольная), вдоль которой гдэны пар смещаются при взаимодействии, и две поперечные оси $j, k$. Рассматриваемая пара ГП помещается в начало системы отсчёта. На неё воздействуют 6 соседних пар $\Gamma \Pi_{j k l}$ (по две на каждой оси), находящихся на расстоянии $r$ от её центра. Их места $j, k, l$ принимают значения \pm 1 . Проекции на ось $l$ мест гдэнов и противогдэнов в паре $\phi_{j k l}^{\Gamma}, \phi_{j k l}^{\Pi}$ расположены симметрично относительно проекции центра пары. Их разность (поляризация) $\xi_{j k l}=\phi_{j k l}^{\Gamma}-\phi_{j k l}^{\Pi}$ мала по сравнению с расстоянием $r$ между ними. Изменение $r$, согласно (16) имеет 2-й порядок малости и будет учтено как нелинейная добавка.

Изменение поляризации определяется потенциалами $\gamma$-воздействия (13) на гдэн $U^{\Gamma}=U_{0}^{\Gamma}+U_{j k l}^{\Gamma}$ и противогдэн $U^{\Pi}=U_{0}^{\Pi}+U_{j k l}^{\Pi}$ в $Г \Pi_{0}$. Они составлены из потенциалов воздействий соседей по паре $U_{0}^{\Gamma}=U_{0}^{\Pi}$ и соседних пар $U_{j k l}^{\Gamma}=U_{j k l}^{\Gamma \Gamma}+U_{j k l}^{\Gamma \Pi}, \quad U_{j k l}^{\Pi}=U_{j k l}^{\Pi \Gamma}+U_{j k l}^{\Pi \Pi}$. Здесь первый верхний значок отмечает гдэн, находящийся под воздействием, а второй - воздействующий.

Потенциал внутреннего взаимодействия в ГП-паре $U_{0}^{\Gamma}=-\gamma e^{-\xi^{2}}$ определяет силы на гдэн $f_{0}^{\Gamma}=-\partial_{\xi} U_{0}^{\Gamma}=-2 \gamma \xi e^{-\xi^{2}} \approx-2 \gamma \xi\left(1-\xi^{2}\right)$ и противогдэн $f_{0}^{\Pi}=-f_{0}^{\Gamma}$. Они разложены до $\xi^{3}$. Остальные силы берутся линейными.

Потенциалы $U_{j k l}^{\Gamma \Gamma}$ и $U_{j k l}^{\Gamma П}-$ близкие по величине и разные по знаку: $U_{j k l}^{\Gamma \Gamma}=U_{j k l}^{\Gamma \Gamma}\left(\xi-\xi_{j k l}\right)=U_{j k l}^{\Gamma \Gamma}(\xi)-\xi_{j k l} \partial_{\xi} U_{j k l}^{\Gamma \Gamma}\left(\xi, \xi_{j k l}=0\right), \quad \partial_{\xi}=\partial / \partial \xi$, $U_{j k l}^{\Gamma \Pi}=U_{j k l}^{\Gamma \Pi}\left(\xi+\xi_{j k l}\right)=-U_{j k l}^{\Gamma \Gamma}(\xi)-\xi_{j k l} \partial_{\xi} U_{j k l}^{\Gamma \Gamma}\left(\xi, \xi_{j k l}=0\right)$, $U_{j k l}^{\Gamma}=U_{j k l}^{\Gamma \Gamma}+U_{j k l}^{\Gamma \Pi}=-2 \xi_{j k l} \partial_{\xi} U_{j k l}^{\Gamma \Gamma}\left(\xi, \xi_{j k l}=0\right)$.

Т. к. место гдэна $\phi^{\Gamma}=\xi / 2$, то на него действует сила $-\partial_{\phi \Gamma} U_{j k l}^{\Gamma}=-2 \partial_{\xi} U_{j k l}^{\Gamma}$. Из симметрии решётки и расположения гдэнов в ГП-паре следует, что в линейном приближении на противогдэн действует противоположная сила. Тогда действующая на смещение $\xi$ сила воздействия пары $\Gamma \Pi_{j k l}$ есть $\xi_{j k l} F_{j k l}$, где

$$
F_{j k l}=8 \partial_{\xi}^{2} U_{j k l}^{\Gamma \Gamma}\left(\xi=\xi_{j k l}=0\right)
$$

является чётной по каждой оси: $F_{1 k l}=F_{-1 k l}, \ldots$

Теперь меняющая поляризацию сила с учётом чётности $F_{j k l}$ есть $f=-4 \gamma \xi\left(1-\xi^{2}\right)+\sum_{j k l} \xi_{j k l} F_{j k l}$, 
$\sum_{j k l} \xi_{j k l} F_{j k l}=\left[\left(\xi_{100}+\xi_{-100}\right) F_{100}+\left(\xi_{010}+\xi_{0-10}\right) F_{010}+\left(\xi_{001}+\xi_{00-1}\right) F_{001}\right]$.

Переходя к разностным производным вдоль каждой оси $i=j, k, l$

$\delta_{i} \xi_{1 / 2}=\xi_{1}-\xi, \delta_{i}^{2} \xi=\delta_{i} \xi_{1 / 2}-\delta_{i} \xi_{-1 / 2}=\left(\xi_{1}-\xi\right)-\left(\xi-\xi_{-1}\right)=\xi_{1}+\xi_{-1}-2 \xi$, получим

$f=-4 \gamma \xi\left(1-\xi^{2}\right)+\left[F_{100}\left(\delta_{j}^{2}+2\right)+F_{010}\left(\delta_{k}^{2}+2\right)+F_{001}\left(\delta_{l}^{2}+2\right)\right] \xi$.

Чтобы найти $F_{j k l}$ используем потенциалы (13)

$U_{100}^{\Gamma \Gamma}=\gamma \exp \left\{-r^{2}-\left(\xi_{100}-\xi\right)^{2} / 4\right\}$, Тогда

$\partial_{\xi} U_{100}^{\Gamma \Gamma}=\gamma\left(\xi_{100}-\xi\right) \exp \left\{-r^{2}-\left(\xi_{100}-\xi\right)^{2} / 4\right\} /\left.2\right|_{\xi_{100}=0}$

$=-\gamma \xi \exp \left\{-r^{2}-\xi^{2} / 4\right\} / 2, \quad F_{100}=-4 \gamma \partial_{\xi}\left[\xi \exp \left\{-r^{2}-\xi^{2} / 4\right\}\right]_{\xi=0}=-4 \gamma e^{-r^{2}}$.

Для другой поперечной оси $F_{010}=F_{100}$.

Для продольной оси

$U_{001}^{\Gamma \Gamma}=\gamma \exp \left\{-\left[r+\left(\xi_{001}-\xi\right) / 2\right]^{2}\right\}$,

$\partial_{\xi} U_{001}^{\Gamma \Gamma}=\left.\gamma\left[r+\left(\xi_{001}-\xi\right) / 2\right] \exp \left\{-\left[r+\left(\xi_{001}-\xi\right) / 2\right]^{2}\right\}\right|_{\xi_{001}=0}$

$=\gamma(r-\xi / 2) \exp \left\{-(r-\xi / 2)^{2}\right\}, \quad F_{001}=8 \gamma \partial_{\xi}\left[(r-\xi / 2) \exp \left\{-(r-\xi / 2)^{2}\right\}\right]_{\xi=0}$ $=8 \gamma\left[-1 / 2+(r-\xi / 2)^{2}\right] \exp \left\{-(r-\xi / 2)^{2}\right\}_{\xi=0}=4 \gamma\left(2 r^{2}-1\right) e^{-r^{2}}=-4 \gamma f_{r}(r)$.

Тогда $f=4 \gamma\left[-\left(1-\xi^{2}\right)-e^{-r^{2}}\left(\delta_{j}^{2}+\delta_{k}^{2}+4\right)+\left(2 r^{2}-1\right) e^{-r^{2}}\left(\delta_{l}^{2}+2\right)\right] \xi$.

Меняя обозначения на $\omega_{\gamma}^{2}=4 \gamma, U=e^{-r^{2}}, f_{r}=\left(1-2 r^{2}\right) U$ из $(14,15)$ и вводя $c_{1}^{2}=\omega_{\gamma}^{2} U, c_{l 1}^{2}=-\omega_{\gamma}^{2} f_{r}=\left(2 r^{2}-1\right) c_{1}^{2}$, получим

$f=\left[-\left(\omega_{\gamma}^{2}+4 c_{1}^{2}-2 c_{l 1}^{2}\right)+\omega_{\gamma}^{2} \xi^{2}-c_{1}^{2}\left(\delta_{j}^{2}+\delta_{k}^{2}\right)+c_{l 1}^{2} \delta_{l}^{2}\right] \xi$.

Найдём влияние изменения расстояния $h$ между ГП-парами продольной оси на поляризацию. Для этого вместо равенства $F_{001}=F_{00-1}=$ $c_{l 1}^{2}(r)$ надо взять $F_{00 \pm 1}=c_{l 1}^{2}\left(r+h_{ \pm 1 / 2}\right) \approx c_{l 1}^{2}(r)+d_{r} c_{l 1}^{2}(r) h_{ \pm 1 / 2}$. Тогда в выражении для силы $f$ слагаемое $\left(\xi_{001}+\xi_{00-1}\right) F_{001}$ меняется на

$\xi_{001} F_{001}+\xi_{00-1} F_{00-1}=\left(\xi_{001}+\xi_{00-1}\right) c_{l 1}^{2}(r)+d_{r} c_{l 1}^{2}(r)\left(\xi_{001} h_{1 / 2}+\xi_{00-1} h_{-1 / 2}\right)$.

Здесь первая скобка приводит к полученной выше формуле $f=\ldots$, а вторая, учитывая гладкость $h$, преобразуется в разностные производные: $\xi_{001} h_{1 / 2}+\xi_{00-1} h_{-1 / 2}=\left[\xi_{001}\left(h_{1}+h_{0}\right)+\xi_{00-1}\left(h_{-1}+h_{0}\right)\right] / 2$ $=\left[\xi_{001} h_{1}+\xi_{00-1} h_{-1}+h_{0}\left(\xi_{001}+\xi_{00-1}\right)\right] / 2$ $=\left[\left(\delta_{l}^{2}+2\right)(\xi h)+h\left(\delta_{l}^{2}+2\right) \xi\right] / 2=\left[\delta_{l}^{2}(\xi h)+h \delta_{l}^{2} \xi+4 \xi h\right] / 2, h \equiv h_{0}$.

Это выражение, умноженное на $d_{r} c_{l 1}^{2}(r)$ добавляется к силе $f$.

Влияние $\xi$-поля поляризации на расстояние между ГП-парами вычисляется из взаимодействия маятников ГП (16) $\ddot{r}=-f_{r r} \xi_{1} \xi_{2}$ (в единицах $2 \gamma)$. Оно состоит из двух одинаковых, но противоположных ускорений пар. Следовательно смещение $a$ выделенной пары ГП при воздействии соседних пар находится из $\ddot{a}=f_{r r} \xi\left(\xi_{001}-\xi_{00-1}\right) / 2=f_{r r} \xi \delta_{l} \xi=f_{r r} \delta_{l} \xi^{2} / 2$, а изменение расстояния между ГП-парами $h=\delta_{l} a$ подчинено уравнению $\ddot{h}=f_{r r} \delta_{l}^{2} \xi^{2} / 2$. Тогда в обычных единицах 
$\ddot{h}=\ddot{r}=\omega_{\gamma}^{2} f_{r r} \delta_{l} \xi^{2} / 4=-d_{r} c_{l 1}^{2}(r) \delta_{l} \xi^{2} / 4$.

Теперь уравнения поляризации и деформации ГП-среды $\ddot{\xi}=\left[-\omega_{0}^{2}-c_{1}^{2}\left(\delta_{j}^{2}+\delta_{k}^{2}\right)+c_{l 1}^{2} \delta_{l}^{2}+\omega_{\gamma}^{2} \xi^{2}\right] \xi+d_{r} c_{l 1}^{2}\left[\delta_{l}^{2}(\xi h)+h \delta_{l}^{2} \xi+4 \xi h\right] / 2$, $\ddot{h}=-d_{r} c_{l 1}^{2} \delta_{l}^{2} \xi^{2} / 4, \quad \omega_{0}^{2}=\omega_{\gamma}^{2}+4 c_{1}^{2}-2 c_{l 1}^{2}, \quad c_{1}^{2}=\omega_{\gamma}^{2} U, \quad c_{l 1}^{2}=\left(2 r^{2}-1\right) c_{1}^{2}$. Здесь $\omega_{0}^{2}-$ квадрат частоты линейного колебания гдэна и противогдэна в ГП-паре с учётом влияния соседних пар, или его удельная внутренняя энергия (внутэн). $\omega_{\gamma}^{2}$ - то же для колебаний в свободной от воздействий паре - маятнике ГП (15). Минус перед $c_{1}^{2}$ означает обратный поперечный перенос возмущения, а плюс перед $c_{l 1}^{2}-$ прямой продольный.

Качественное различие поперечного и продольного переносов определяется особенностями взаимодействий гдэнов по этим направлениям. Вдоль продольной оси соседние ГП-пары расположены достаточно далеко - на расстояние $r$ между ними. Смещённый гдэн одной из них притягивает противогдэн и отталкивает гдэн другой пары, создавая в ней смещение, подобное своему, и передаёт туда часть своей энергии. Образуется прямой перенос. Вдоль поперечных осей проекции смещений гдэнов на ось поляризации совмещаются. Здесь смещённый гдэн также притягивает противогдэн и отталкивает гдэн другой пары, но вызывает при этом смещение, обратное своему. Направления поляризации у соседних по поперечным осям пар становятся противоположными, что даёт ГП-среде наименьшую энергию. Такое строение ГП-среды учитывается изменением знака перед $c_{1}^{2}$ :

$$
\begin{aligned}
& \ddot{\xi}=\left[-\omega_{m}^{2}+c_{1}^{2}\left(\delta_{j}^{2}+\delta_{k}^{2}\right)+c_{l 1}^{2} \delta_{l}^{2}+\omega_{\gamma}^{2} \xi^{2}\right] \xi+d_{r} c_{l 1}^{2}\left[\delta_{l}^{2}(\xi h)+h \delta_{l}^{2} \xi+4 \xi h\right] / 2, \\
& \ddot{h}=-d_{r} c_{l 1}^{2} \delta_{l}^{2} \xi^{2} / 4, \quad \omega_{m}^{2}=\omega_{\gamma}^{2}-4 c_{1}^{2}-2 c_{l 1}^{2}, c_{1}^{2}=\omega_{\gamma}^{2} U, c_{l 1}^{2}=\left(2 r^{2}-1\right) c_{1}^{2} .
\end{aligned}
$$

\section{Две решётки куба.}

Чтобы приблизиться к симметрии сферы с сохранением точечных взаимодействий, к решётке куба добавляется такая же подрешётка из восьми ГП-пар на расстоянии $\rho=r / \sqrt{3}$ от выделенной пары. Это должно привести к уменьшению поперечной скорости, увеличению продольной и изменению частоты $\omega_{m}$ колебаний в паре. Влияние подрешётки вызывает добавочную силу $f_{a}=\sum_{J K L} \xi_{J K L} F_{J K L}$, где $J, K, L= \pm 1$ в единицах $\rho$. С учётом чётности по всем осям $F_{J K L}=F_{111}$ и $f_{a}=F_{111} \sum_{J K L} \xi_{J K L}$

$=F_{111}\left[\left(\xi_{111}+\xi_{-111}\right)+\left(\xi_{1-11}+\xi_{-1-11}\right)+\left(\xi_{11-1}+\xi_{-11-1}\right)+\left(\xi_{1-1-1}+\xi_{-1-1-1}\right)\right]$

$=F_{111}\left(\delta_{J}^{2}+2\right)\left[\left(\xi_{011}+\xi_{0-11}\right)+\left(\xi_{01-1}+\xi_{0-1-1}\right)\right]$

$=F_{111}\left(\delta_{J}^{2}+2\right)\left(\delta_{K}^{2}+2\right)\left(\xi_{001}+\xi_{00-1}\right)=F_{111}\left(\delta_{J}^{2}+2\right)\left(\delta_{K}^{2}+2\right)\left(\delta_{L}^{2}+2\right) \xi$. 
Оставляя только вторые производные получим

$f_{a}=4 F_{111}\left(\delta_{J}^{2}+\delta_{K}^{2}+\delta_{L}^{2}+2\right) \xi$.

Потенциал воздействия (13) между гдэнами

$U_{111}^{\Gamma \Gamma}=\gamma \exp \left\{-2 \rho^{2}-\left[\rho+\left(\xi_{111}-\xi\right) / 2\right]^{2}\right\}$,

$\partial_{\xi} U_{111}^{\Gamma \Gamma}\left(\xi_{111}=0\right)=\left.\gamma\left[\rho+\left(\xi_{111}-\xi\right) / 2\right] \exp \left\{-2 \rho^{2}-\left[\rho+\left(\xi_{111}-\xi\right) / 2\right]^{2}\right\}\right|_{\xi_{111}=0}$ $=\gamma(\rho-\xi / 2) \exp \left\{-2 \rho^{2}-(\rho-\xi / 2)^{2}\right\}$,

$\partial_{\xi}^{2} U_{111}^{\Gamma \Gamma}\left(\xi=\xi_{111}=0\right)=\left.\gamma\left[-1 / 2+(\rho-\xi / 2)^{2}\right] \exp \left\{-2 \rho^{2}-(\rho-\xi / 2)^{2}\right\}\right|_{\xi=0}$

$=\gamma\left(2 \rho^{2}-1\right) e^{-3 \rho^{2}} / 2$,

$F_{111}=8 \partial_{\xi}^{2} U_{111}^{\Gamma \Gamma}\left(\xi=\xi_{111}=0\right)=4 \gamma\left(2 \rho^{2}-1\right) e^{-3 \rho^{2}}$.

Тогда $f_{a}=16 \gamma\left(2 \rho^{2}-1\right) e^{-3 \rho^{2}}\left(\delta_{J}^{2}+\delta_{K}^{2}+\delta_{L}^{2}+2\right) \xi$.

Переходя к обозначению мест ГП-пар в единицах $r$ запишем $\{J, K, L\}=\sqrt{3}\{j, k, l\}, \delta_{J}^{2}=\delta_{j}^{2} / 3, \ldots, \rho^{2}=r^{2} / 3$,

$f_{a}=(16 / 9) \gamma\left(2 r^{2}-3\right) e^{-r^{2}}\left(\delta_{j}^{2}+\delta_{k}^{2}+\delta_{l}^{2}+6\right) \xi$.

Вводя $\omega_{\gamma}^{2}=4 \gamma, U=e^{-r^{2}}$ из $(14,15)$ и $c_{a}^{2}=4\left(2 r^{2}-3\right) c_{1}^{2} / 9$ получим $f_{a}=c_{a}^{2}\left(\delta_{j}^{2}+\delta_{k}^{2}+\delta_{l}^{2}+6\right) \xi$.

Теперь суммарное ускорение, возникающее при воздействии соседних ГП-пар двух решёток куба

$\ddot{\xi}=\left[-\omega_{\gamma}^{2}-4 c_{1}^{2}+2 c_{l 1}^{2}+6 c_{a}^{2}+\omega_{\gamma}^{2} \xi^{2}-c_{1}^{2}\left(\delta_{j}^{2}+\delta_{k}^{2}\right)+c_{l 1}^{2} \delta_{l}^{2}+c_{a}^{2}\left(\delta_{j}^{2}+\delta_{k}^{2}+\delta_{l}^{2}\right)\right] \xi$. Если здесь $c_{1}^{2}>c_{a}^{2}$, то поперечный перенос будет обратным, в противном случае прямым. Оба случая можно объединить и представить прямым поперечным переносом, вводя

$c_{2}^{2}=\left|c_{a}^{2}-c_{1}^{2}\right|=\left|4\left(2 r^{2}-3\right) / 9-1\right| c_{1}^{2}=\left|8 r^{2}-21\right| c_{1}^{2} / 9$,

$c_{l 2}^{2}=c_{l 1}^{2}+c_{a}^{2}=\left[\left(2 r^{2}-1\right)+4\left(2 r^{2}-3\right) / 9\right] c_{1}^{2}=\left(26 r^{2}-21\right) c_{1}^{2} / 9$.

Тогда уравнение поляризации в двойной решётке куба

$$
\begin{aligned}
& \ddot{\xi}=\left[-\omega_{m}^{2}+\omega_{\gamma}^{2} \xi^{2}+c_{2}^{2}\left(\delta_{j}^{2}+\delta_{k}^{2}\right)+c_{l 2}^{2} \delta_{l}^{2}\right] \xi \\
& \omega_{m}^{2}=\omega_{\gamma}^{2}-4 c_{2}^{2}-2 c_{l 2}^{2}, \quad c_{2}^{2}=\left|21-8 r^{2}\right| c_{1}^{2} / 9, \quad c_{l 2}^{2}=\left(26 r^{2}-21\right) c_{1}^{2} / 9 .
\end{aligned}
$$

\section{Непрерывное распределение по сфере.}

Полностью изотропную модель ГП-среды получим, если все влияющие на выделенную ГП-пару соседи расположены вокруг неё на сфере радиуса $r$. Она может содержать $N \sim 10 \div 15$ ГП-пар, что приблизительно соответствует числу соседей при плотной упаковке шаров. Их точечное расположение на сфере заменяется непрерывным с плотностью $N /\left(4 \pi r^{2}\right)$.

На направленную вдоль оси $l$ поляризацию $\xi$ пары $Г \Pi_{0}$, находящейся в начале системы отсчёта, действует ГП-слой с поляризацией $\xi(\theta, \chi)$. 
Так же как для решётки куба изменение поляризации определяется потенциалами $\gamma$-воздействия (13), но вместо суммы берётся интеграл по поверхности сферы.

Действующая на $\xi$ сила

$f=-4 \gamma \xi\left(1-\xi^{2}\right)+\int d s \xi(\theta, \chi) F(\theta, \chi)$

записана в линейном по $\xi / r$ приближении, соответствующим (18), где

$F(\theta, \chi)=8 \partial_{\xi}^{2} U^{\Gamma \Gamma}(\theta, \chi, \xi=\xi(\theta, \chi)=0)$.

Здесь $U^{\Gamma \Gamma}(\theta, \chi)=g e^{-\rho^{2}}, g=N \gamma /\left(4 \pi r^{2}\right), \rho^{2}=r^{2}+b^{2}+2 r b \cos \theta$,

$b=\xi(\theta, \chi)-\xi, d s=r^{2} \sin \theta d \theta d \chi, \theta-$ угол с осью $l, \chi-$ угол в плоскости $j, k$, который отсчитывается от оси $j$. В этих переменных

$F=\left.8 g \partial_{b}^{2} e^{-\rho^{2}}\right|_{b=0}=-16 g \partial_{b}\left[(b+r \cos \theta) e^{-\rho^{2}}\right]_{b=0}$

$=-\left.16 g\left[1-2(b+r \cos \theta)^{2}\right] e^{-\rho^{2}}\right|_{b=0}=16 g\left(2 r^{2} \cos ^{2} \theta-1\right) e^{-r^{2}}$.

Обозначая $c=\cos \theta, r_{c}=r c, r_{s}=r \sin \theta, h_{c}=r_{s} c, h_{s}=r_{s} \sin \theta$, запишем

$f=-4 \gamma \xi\left(1-\xi^{2}\right)+16 g r^{2} e^{-r^{2}} \int_{-1}^{1}\left(2 r_{c}^{2}-1\right) d c \int_{-\pi}^{\pi} d \chi \xi(\theta, \chi)$.

(Видно, что при $r_{c}^{2}=1 / 2$ плотность силы меняет знак. Это приводит к качественно разному взаимодействию со слоем вблизи поперечных и продольной осей.)

$$
\begin{aligned}
& \int_{-\pi}^{\pi} \xi(\theta, \chi) d \chi=\int_{-\pi / 2}^{\pi / 2}[\xi(\theta, \chi)+\xi(\theta, \pi-\chi)] d \chi=\int_{-\pi / 2}^{\pi / 2}\left[\left(h_{c} / r\right)^{2} \delta_{j}^{2}+2\right] \xi_{k}(\theta, \chi) d \chi \\
& =\int_{0}^{\pi / 2}\left[\left(h_{c} / r\right)^{2} \delta_{j}^{2}+2\right]\left[\xi_{k}(\theta, \chi)+\xi_{k}(\theta,-\chi)\right] d \chi \\
& =\int_{0}^{\pi / 2}\left[\left(h_{c} / r\right)^{2} \delta_{j}^{2}+2\right]\left[\left(h_{s} / r\right)^{2} \delta_{k}^{2}+2\right] d \chi \xi_{l}(\theta) \\
& \approx 2 \int_{0}^{\pi / 2}\left[\left(h_{c} / r\right)^{2} \delta_{j}^{2}+\left(h_{s} / r\right)^{2} \delta_{k}^{2}+2\right] d \chi \xi_{l}(\theta)=\pi\left[\left(r_{s}^{2} / 2 r^{2}\right)\left(\delta_{j}^{2}+\delta_{k}^{2}\right)+2\right] \xi_{l}(\theta) .
\end{aligned}
$$

Здесь $\xi_{k}(\theta, \chi)=[\xi(\theta, \chi)+\xi(\theta, \pi-\chi)] / 2-$ среднее значение смещения на сфере, перенесённое на ось $k$, что необходимо для введения второй разностной производной $\delta_{j}^{2} \cdot \xi_{l}(\theta)=\left[\xi_{k}(\theta, \chi)+\xi_{k}(\theta,-\chi)\right] / 2-$ то же для $\delta_{k}^{2}$. Производные выше 2-го порядка не учитываются.

Теперь, обозначая $\delta_{t}^{2}=\delta_{j}^{2}+\delta_{k}^{2}$, запишем

$$
\begin{aligned}
& \int_{-1}^{1}\left(2 r_{c}^{2}-1\right) d c \int_{-\pi}^{\pi} d \chi \xi(\theta, \chi)=\pi \int_{-1}^{1}\left(2 r_{c}^{2}-1\right)\left[\left(\sin ^{2} \theta \delta_{t}^{2} / 2+2\right] \xi_{l}(\theta) d c,\right. \\
& =\pi \int_{0}^{1}\left(2 r_{c}^{2}-1\right)\left[\left(1-c^{2}\right) \delta_{t}^{2} / 2+2\right]\left[\xi_{l}(\theta)+\xi_{l}(\pi-\theta)\right] d c
\end{aligned}
$$




$$
\begin{aligned}
& =\pi \xi \int_{0}^{1}\left(2 r_{c}^{2}-1\right)\left[\left(1-c^{2}\right) \delta_{t}^{2} / 2+2\right]\left[\left(r_{c} / r\right)^{2} \delta_{l}^{2}+2\right] d c \\
& \approx \pi \xi \int_{0}^{1}\left(2 r^{2} c^{2}-1\right)\left[\left(1-c^{2}\right) \delta_{t}^{2}+2 c^{2} \delta_{l}^{2}+4\right] d c . \\
& \int_{0}^{1} \ldots=\int_{0}^{1}\left(2 r^{2} c^{2}-1\right)\left[\delta_{t}^{2}+4+\left(2 \delta_{l}^{2}-\delta_{t}^{2}\right) c^{2}\right] d c \\
& =2 r^{2}\left[\left(\delta_{t}^{2}+4\right) / 3+\left(2 \delta_{l}^{2}-\delta_{t}^{2}\right) / 5\right]-\delta_{t}^{2}-4-\left(2 \delta_{l}^{2}-\delta_{t}^{2}\right) / 3 \\
& =4\left(2 r^{2} / 3-1\right)+\left(4 r^{2} / 15-2 / 3\right) \delta_{t}^{2}+\left(4 r^{2} / 5-2 / 3\right) \delta_{l}^{2} .
\end{aligned}
$$

Окончательно сила

$f=-4 \gamma \xi\left(1-\xi^{2}\right)+8 N \gamma e^{-r^{2}}\left[2\left(2 r^{2} / 3-1\right)+\left(2 r^{2}-5\right) \delta_{t}^{2} / 15+\left(6 r^{2}-5\right) \delta_{l}^{2} / 15\right]$,

Отсюда следует уравнение для поляризации, которое подобно (20) не имеет обратного поперечного переноса,

$$
\begin{aligned}
& \ddot{\xi}=\left[-\omega_{m}^{2}-\omega_{\gamma}^{2} \xi^{2}+c_{3}^{2}\left(\delta_{j}^{2}+\delta_{k}^{2}\right)+c_{l 3}^{2} \delta_{l}^{2}\right] \xi, \quad \omega_{m}^{2}=\omega_{\gamma}^{2}-4 c_{3}^{2}-2 c_{l 3}^{2}, \\
& c_{3}^{2}=2(N / 15) \omega_{\gamma}^{2}\left|2 r^{2}-5\right| c_{1}^{2}, \quad c_{l 3}^{2}=2(N / 15)\left(6 r^{2}-5\right) c_{1}^{2}, \quad N \sim 10 \div 15 .
\end{aligned}
$$

Уравнения поляризации (19-21) одинаковые, но для разных моделей ГП-среды имеют разные скорости переноса. Общие уравнения с учётом изменения расстояния между ГП-парами имеют вид

$$
\begin{aligned}
& \ddot{\xi}=\left[-\omega_{m}^{2}+c^{2}\left(\delta_{j}^{2}+\delta_{k}^{2}\right)+c_{l}^{2} \delta_{l}^{2}+\omega_{\gamma}^{2} \xi^{2}\right] \xi+d_{r} c_{l}^{2}\left[\delta_{l}^{2}(\xi h)+h \delta_{l}^{2} \xi+4 \xi h\right] / 2, \\
& \ddot{h}=-d_{r} c_{l}^{2} \delta_{l}^{2} \xi^{2} / 4, \quad \omega_{m}^{2}=\omega_{\gamma}^{2}-4 c^{2}-2 c_{l}^{2}
\end{aligned}
$$

где $c^{2}=c_{i}^{2}, c_{l}^{2}=c_{l i}^{2}, i=1,2,3:$

$c_{1}^{2}=\omega_{\gamma}^{2} e^{-r^{2}}, \quad c_{l 1}^{2}=\left(2 r^{2}-1\right) c_{1}^{2}-$ простая решётка куба,

$c_{2}^{2}=\left|21-8 r^{2}\right| c_{1}^{2} / 9, \quad c_{l 2}^{2}=\left(26 r^{2}-21\right) c_{1}^{2} / 9$ - двойная решётка куба,

$c_{3}^{2}=2(N / 15)\left|2 r^{2}-5\right| c_{1}^{2}, \quad c_{l 3}^{2}=2(N / 15)\left(6 r^{2}-5\right) c_{1}^{2}$ - cфера.

Приближение сплошной среды получается заменой разностных производных на частные для непрерывных функций $\xi, h$

$$
\begin{aligned}
& \ddot{\xi}=\left[-\omega_{m}^{2}+\omega_{\gamma}^{2} \xi^{2}+c^{2}\left(\partial_{j}^{2}+\partial_{k}^{2}\right)+c_{l}^{2} \partial_{l}^{2}\right] \xi+d_{r} c_{l}^{2}\left[\partial_{l}^{2}(\xi h)+h \partial_{l}^{2} \xi+4 \xi h\right] / 2, \\
& \ddot{h}=-d_{r} c_{l}^{2} \partial_{l}^{2} \xi^{2}, \quad \omega_{m}^{2}=\omega_{\gamma}^{2}-4 c^{2}-2 c_{l}^{2} .
\end{aligned}
$$

Теперь строение ГП-среды проявляется только через различия в значениях скоростей переноса возмущений.

Чтобы найти векторное уравнение поляризации введём вектор $\boldsymbol{\xi}=\left\{\xi_{j}, \xi_{k}, \xi_{l},\right\}$ и учтём различие поперечных $\boldsymbol{\xi}_{t}$ и продольных $\boldsymbol{\xi}_{l}$ полей: $\boldsymbol{\xi}=\boldsymbol{\xi}_{t}+\boldsymbol{\xi}_{l}, \operatorname{div} \boldsymbol{\xi}_{t}=0, \operatorname{rot} \boldsymbol{\xi}_{l}=0$. В лапласиане $\Delta=\operatorname{grad} \operatorname{div}-\operatorname{rot} \operatorname{rot}$ 
выделяются продольная $\Delta_{l}=\operatorname{grad} \operatorname{div}$ и поперечная $\Delta_{t}=-\operatorname{rot} \operatorname{rot}$ части. Тогда из (23) следует

$$
\ddot{\boldsymbol{\xi}}+\omega_{m}^{2} \boldsymbol{\xi}=c^{2} \Delta_{t} \boldsymbol{\xi}+c_{l}^{2} \Delta_{l} \boldsymbol{\xi}+\omega_{\gamma}^{2} \xi^{2} \boldsymbol{\xi} .
$$

Левая часть уравнения поляризации описывает колебания в ГП-паре, а правая - их перенос и влияние нелинейности. Его разложим на отдельные уравнения Клейна-Фока-Гордона (урКФГ) [13]-[15] для поперечных $\boldsymbol{\xi}_{t}$ и продольных $\boldsymbol{\xi}_{l}$ полей, связанных только нелинейностью

$$
\ddot{\boldsymbol{\xi}_{t, l}}+\omega_{m}^{2} \boldsymbol{\xi}_{t, l}=c_{t, l}^{2} \Delta_{t, l} \boldsymbol{\xi}_{t, l}+\omega_{\gamma}^{2} \xi^{2} \boldsymbol{\xi}_{t, l} .
$$

\section{Волны поляризации}

Дисперсионное уравнение (диспур) волн в решётке выводится из линейной части уравнения (22) после подстановки $\xi$ в виде гармоники $\xi(\mathbf{x}, t)=R e[\psi \exp \{-i \omega t+i \mathbf{q} \mathbf{x}\}]$, где $\mathbf{q}=\left\{q_{j}, q_{k}, q_{l}\right\}-$ волновой вектор, $\mathbf{x}=\{j, k, l\}-$ координата в номерах ГП-пар. Тогда из $\ddot{\xi}=-\omega^{2} \xi$,

$\delta_{\mathbf{x}}^{2} \xi=\xi_{\mathbf{x}+1}+\xi_{\mathbf{x}-1}-2 \xi=\left(e^{i \mathbf{q}}+e^{-i \mathbf{q}}-2\right) \xi=2(\cos \mathbf{q}-1) \xi=-4 \xi \sin ^{2}(\mathbf{q} / 2)$ следуют диспур $\xi$-волн и вектор групповой скорости $d_{\mathbf{q}} \omega=\mathbf{V}=\left\{V_{j}, V_{k}, V_{l}\right\}$

$$
\begin{gathered}
\omega^{2}=\omega_{m}^{2}+4 c^{2}\left[\sin ^{2}\left(q_{j} / 2\right)+\sin ^{2}\left(q_{k} / 2\right)\right]+4 c_{l}^{2} \sin ^{2}\left(q_{l} / 2\right), \\
\omega_{m}^{2}=\omega_{\gamma}^{2}-4 c^{2}-2 c_{l}^{2}, \quad V_{j, k}=\left(c^{2} / \omega\right) \sin q_{j, k}, \quad V_{l}=\left(c_{l}^{2} / \omega\right) \sin q_{l} .
\end{gathered}
$$

Из него в приближении сплошной среды следует

$$
\omega^{2}=\omega_{m}^{2}+c^{2}\left(q_{j}^{2}+q_{k}^{2}\right)+c_{l}^{2} q_{l}^{2}, \quad V_{j, k}=\left(c^{2} / \omega\right) q_{j, k}, \quad V_{l}=\left(c_{l}^{2} / \omega\right) q_{l}
$$

где $\mathbf{x}=\{j, k, l\}$ теперь непрерывные координаты в единицах расстояния между ГП-парами. После разложения на отдельные уравнения для поперечных $\boldsymbol{\xi}_{t}$ и продольных $\boldsymbol{\xi}_{l}$ волн получим

$$
\omega_{t, l}^{2}=\omega_{m}^{2}+c_{t, l}^{2} q_{t, l}^{2}, \quad V_{t, l}=c_{t, l}^{2} q_{t, l} / \omega_{t, l}, \quad c_{t} \equiv c .
$$

Квадрат частоты (удельная внутэн) колебаний в ГП-паре $\omega_{m}^{2}$ определяет распространение волн и состояние ГП-среды:

Если $\omega_{m}^{2}>0$, то в волне притяжение гдэна $\Gamma^{3}$ и противогдэна $\Pi^{3}$ ГП-пары преобладает над притяжением соседних пар. При этом часть энергии волны расходуется на колебания в паре, а остальное передаётся 
соседям. Волна затухает с расстоянием и называется массивной. Т. к. $\omega^{2}>0$, то постоянное $\xi$-поле отсутствует. ГП-среда ведёт себя подобно твёрдому телу - ГП-твердь.

Если $\omega_{m}^{2}=0$, то притяжение $\Gamma^{3}$ и $\Pi^{3}$ в паре уравновешивается притяжением соседей. Образуется безразличное состояние - граница распада пары. Вся энергия волны заключена в переносе - волна становится безмассовой. Появляется возможность существования постоянного $\xi$-поля. Условие $\omega_{m}^{2}=0$ даёт граничное расстояние $r_{0}$ между ГП-парами.

Если $\omega_{m}^{2}<0$, но немного, то разрушение некоторых ГП-пар и последующая перестройка ГП-среды увеличивают $r$, делая границу распада устойчивой к малому уменьшению расстояния между парами. Образуется относительно небольшое количество свободных гдэнов и противогдэнов, которые становятся основами элементарных частиц (см. "Частицы"). Отклонение $r_{0}-r$ определяет плотность числа частиц во вселенной. Т. к. она много меньше плотности ГП-среды, то $r_{0}-r \ll r_{0}$.

Если $\omega_{m}^{2}<0$, то преобладает притяжение соседних ГП-пар. Даже в отсутствие волн такая неполяризованная среда неустойчива. Любое малое возмущение разрушает ГП-пары. Из гдэнов и противогдэнов образуется подобная плазме среда с тепловой энергией (энергия движения на гдэн) $\left|\omega_{m}^{2}\right| / 2-$ ГП-плазма. Она не годится для образования в ней упорядоченного мира.

Безмассовая волна описывается уравнениями, которые следуют из $(26,28)$ при $\omega_{m}^{2}=0$ :

$$
\begin{aligned}
& \ddot{\xi}_{t}=\left[c^{2}\left(\delta_{j}^{2}+\delta_{k}^{2}\right)+\omega_{\gamma}^{2} \xi^{2}\right] \xi_{t}, \quad \omega_{t}^{2}=4 c^{2}\left[\sin ^{2}\left(q_{j} / 2\right)+\sin ^{2}\left(q_{k} / 2\right)\right], \\
& \ddot{\xi}_{l}=\left(c_{l}^{2} \delta_{l}^{2}+\omega_{\gamma}^{2} \xi^{2}\right) \xi_{l}, \quad \omega_{l}^{2}=4 c_{l}^{2} \sin ^{2}\left(q_{l} / 2\right), \\
& V_{j, k}=\sin \left(q_{j, k}\right) / M_{t}, \quad V_{l}=\sin \left(q_{l}\right) / M_{l} .
\end{aligned}
$$

Здесь $M_{t}=\omega_{t} / c^{2}$ и $M_{l}=\omega_{l} / c_{l}^{2}$ понимаются как массы движущихся квантов соответствующих волн, имеющих частоты $\omega_{t}$, и $\omega_{l}$. Они связывают скорости $\boldsymbol{V}=\left\{V_{j}, V_{k}, V_{l}\right\}$ и импульсы $\boldsymbol{q}=\left\{q_{j}, q_{k}, q_{l}\right\}$ квантов. Массы покоя $m_{t}=\omega_{m} / c^{2}, m_{l}=\omega_{m} / c_{l}^{2}$ равны нулю.

Скорости волн не постоянны

$$
V_{t}^{2}=V_{j}^{2}+V_{k}^{2}=\frac{c^{2}\left(\sin ^{2} q_{j}+\sin ^{2} q_{k}\right)}{4\left[\sin ^{2}\left(q_{j} / 2\right)+\sin ^{2}\left(q_{k} / 2\right)\right]}, \quad V_{l}=c_{l} \cos \left(q_{l} / 2\right) .
$$

Они меняются от $c$ или $c_{l}$ при $q_{j}=q_{k}=0$ или $q_{l}=0$ до $c / \sqrt{2}$ или $c_{l} / \sqrt{2}$ при $q_{j}=q_{k}= \pm \pi / 2$ или $q_{l}= \pm \pi / 2$. 
В приближении сплошной среды из $(25,29)$ следуют векторные уравнения с постоянными скоростями волн

$$
\begin{aligned}
& \ddot{\boldsymbol{\xi}}_{t}=-c^{2} \operatorname{rot} \operatorname{rot} \boldsymbol{\xi}_{t}+\omega_{\gamma}^{2} \xi^{2} \boldsymbol{\xi}_{t}, \operatorname{div} \boldsymbol{\xi}_{t}=0, \omega_{t}=c q_{t}, V_{t}=c, \\
& \ddot{\boldsymbol{\xi}}_{l}=c_{l}^{2} \operatorname{grad} \operatorname{div} \boldsymbol{\xi}_{l}+\omega_{\gamma}^{2} \xi^{2} \boldsymbol{\xi}_{l}, \operatorname{rot} \operatorname{rot} \boldsymbol{\xi}_{l}=0, \omega_{l}=c_{l} q_{l}, V_{l}=c_{l} .
\end{aligned}
$$

Постоянное поле поляризации может существовать лишь вместе с безмассовой $\xi$-волной, т. к. только в ней достижима нулевая частота.

\section{Электрическое поле}

Поле поляризации вокруг гдэна. Пусть в ГП-среде посередине между ГП-парами находится дополнительный гдэн $\Gamma^{3}$. Его потенциальное поле (13) действует на ближайшие к нему пары и создаёт в среде возмущённую гдэном область. При этом основным будет первый член $U_{\gamma}$ разложения потенциала (13). Под его влиянием ближайшие к $\Gamma^{3}$ пары поляризуются, создавая граничные условия для поля поляризации среды. Вдали от $\Gamma^{3}$ при $\omega_{m}=0$ образуется постоянное радиальное поле $\xi(R)$, где $R$ - расстояние от центра $\Gamma^{3}$. В приближении сплошной среды оно находится из уравнения (31): $\operatorname{grad} \operatorname{div} \boldsymbol{\xi}_{l}=d_{R}\left[R^{-2} d_{R}\left(R^{2} \xi\right)\right]=0$. Его убывающее с расстоянием $R$ решение есть $\xi(R)=e / R^{2}$. Конечный внутренний радиус поля обуславливает его конечную энергию.

\section{Уравнения Максвелла}

В приближении сплошной среды для безмассовой поперечной волны $\boldsymbol{\xi}=\boldsymbol{\xi}_{t}$ линейная часть уравнения (31) есть $\ddot{\xi}=-c^{2} \operatorname{rot} \operatorname{rot} \boldsymbol{\xi}$. Т. к. $\dot{\boldsymbol{\xi}}$ тоже поперечный вектор, то его можно записать как ротор векторной функции $\dot{\boldsymbol{\xi}}=c \operatorname{rot} \mathbf{b}$. Тогда $\ddot{\xi}=c \operatorname{rot} \dot{\mathbf{b}}, \quad \dot{\mathbf{b}}=-c \operatorname{rot} \boldsymbol{\xi}, \quad \operatorname{div} \dot{\mathbf{b}}=0$. Отсутствие причин для введения $b$-заряда позволяет записать $\operatorname{div} \mathbf{b}=0$. Полученная система уравнений

$\dot{\boldsymbol{\xi}}=c \operatorname{rot} \mathbf{b}, \quad \dot{\mathbf{b}}=-c \operatorname{rot} \boldsymbol{\xi}, \quad \operatorname{div} \boldsymbol{\xi}=\operatorname{div} \mathbf{b}=0$

совпадает с уравнениями Максвелла для электромагнитного поля в отсутствие зарядов и токов.

Зарядами в ГП-среде являются отдельные гдэны или противогдэны с полем $\xi(R)=e / R^{2}$. Это поле Кулона по теореме о дивергенции ГауссаОстроградского приводит к закону Гаусса: Для малой сферы радиуса $R$ интеграл по её объёму от $\operatorname{div} \boldsymbol{\xi}$ равен интегралу по её поверхности от $\boldsymbol{\xi} \boldsymbol{n}$

$$
\frac{4}{3} \pi R^{3} \operatorname{div} \boldsymbol{\xi}=\int \operatorname{div} \boldsymbol{\xi} d^{3} x=\oint \boldsymbol{\xi} \boldsymbol{n} d^{2} x=4 \pi R^{2} \boldsymbol{\xi}(R)=4 \pi e=4 \pi \frac{4 \pi}{3} \rho R^{3},
$$


где $\mathbf{n}-$ внешняя нормаль сферы, $\rho$ объёмная плотность заряда в сфере. Отсюда $\operatorname{div} \boldsymbol{\xi}=4 \pi \rho$. Из уравнения непрерывности $\dot{\rho}+\operatorname{div} \mathbf{j}=0$, где введена плотность тока $\mathbf{j}$, следует $\operatorname{div} \dot{\boldsymbol{\xi}}=4 \pi \dot{\rho}=-4 \pi \operatorname{div} \mathbf{j}$ и $\dot{\boldsymbol{\xi}}=-4 \pi \mathbf{j}$.

В линейном приближении поля складываются и образуется система уравнений

$$
\dot{\boldsymbol{\xi}}=c \operatorname{rot} \mathbf{b}-4 \pi \mathbf{j}, \quad \operatorname{div} \boldsymbol{\xi}=4 \pi \rho, \quad \dot{\mathbf{b}}=-c \operatorname{rot} \boldsymbol{\xi}, \quad \operatorname{div} \mathbf{b}=0 .
$$

Это уравнения Максвелла для напряжённости электрического поля $\boldsymbol{\xi}$ и индукции магнитного поля $\mathbf{b}$ в среде с электрическими зарядами и токами, имеющими объёмные плотности $\rho$ и $\mathbf{j}$.

Итак напряжённость электрического поля можно отождествить с частью поляризации ГП-пар, включающей постоянное $\xi$-поле гдэнов и поперечную безмассовую волну. Магнитное поле характеризует изменение $\xi$-поля во времени и не является основополагающим. Его можно без ущерба для смысла убрать из названия поля, оставив только прилагательное "электрический": электрическое поле (электрополе) и электрическая волна (электроволна). Вопрос о продольной части $\xi$-поля рассмотрен в следующем разделе.

Электроволна есть безмассовая поперечная $\xi$-волна. Её длина волны $\lambda>4$ в единицах расстояний $r_{0}$ между парами. Если $r_{0} \sim 10^{-22}$ м опытный размер электрона [11], то $\lambda>4 \cdot 10^{-22}$ м, а частота волны менее $10^{30}$ Гц (для меньших $r_{0}$ граничные параметры волн соответственно изменяться). Её групповая скорость (30) меняется от скорости света $c$ для длинных волн до $c / 2$ при $\lambda=4$. Но длинноволновой предел сплошной среды (30), в котором она постоянна, охватывает все наблюдаемые волны.

Надшумные гдэны имеют распределение (12) с пространственной дисперсией $\left\langle\dot{\phi}^{2}\right\rangle=3 / 2$ и шириной $\left\langle\dot{\phi}^{2}\right\rangle^{1 / 2} \sim 1$. Её удвоенное значение даёт дисперсию поляризации ГП-пары, или неустранимый шум поляризации $\delta \xi \sim 2$, намного превышающий величину электрополя. Но этот шумный фон незаметен, поскольку противоположные флуктуации взаимно уничтожаются усреднением при наблюдении за времена много большие их длительностей.

Наш мир может существовать если расстояния между ГП-парами $r=r_{0}$ выдержаны с огромной точностью, обеспечивающей лишь геометрическое пространственное ослабление поляризации. В ГП-тверди $\left(r>r_{0}\right)$ волны становятся массивными и быстро затухают с удалением от источника, а постоянное поле отсутствует. В ГП-плазме $\left(r<r_{0}\right)$ 
устойчивая поляризация не возможна. Тонкая граница между ними есть среда нашего мира. Она, как носитель электрополя, подобна гипотетическому эфиру Декарта. Для неё можно оставить это название эфир Э, а её элементы назвать эфироны э $=\Gamma^{3} \gamma \Pi^{3}$ при $r=r_{0}$.

Трудно представить, чтобы такие особые условия могли постоянно поддерживаться во всём пространстве нашей независимой вселенной. Более естественно предположить, что эфир есть 3-мерная поверхность в 4-мерной среде, разделяющая ГП-плазму и ГП-твердь. Такая поверхность может возникать, изменятся и пропадать, сохраняя своё качество. Сравнение с эволюцией нашей вселенной приводит к предположению о фазовом переходе 4-среды из плазмы в твердь, который начинается с точечного зародыша и продолжается увеличением граничной поверхности до смены состояния всей 4-среды или обратного её движения. Для поверхности перехода естественно иметь меняющуюся во времени кривизну, которая может быть представлена космологической постоянной [12] в уравнениях гравитации Эйнштейна.

\section{Массивные поля}

Безмассовая волна существует, если расстояние $r$ между эфиронами не превышает критического значения, обеспечивающего нулевую частоту $\omega_{m}$ колебаний гдэнов в эфироне. Присутствие в эфире дополнительного гдэна $\Gamma^{3}$ (или $\Pi^{3}$ ) меняет $r$. В соседнем эфироне он притягивает противогдэн и отталкивает гдэн. Если сила их взаимодействия убывает с расстоянием, то притяжение сильнее отталкивания. Ближайшие эфироны сдвигаются к гдэну, растягивая за собой эфир и образуя область разрежения, в которой $\omega_{m}^{2}>0$.

В ней могут существовать поля стационарных поперечных и продольных (относительно радиуса) колебаний $\xi_{t, l}(R, t)=R e\left[\psi_{t, l}(R) e^{-i \omega_{m}(R) t}\right]$, где $R$ - расстояние от гдэна. Постоянное радиальное поле в этой области существовать не может, т. к. $\omega_{m}$ определяет нижний порог частоты. На больших расстояниях, где $\omega_{m}=0$ остаётся кулоново поле.

Величины полей вблизи гдэна $\Gamma^{3}$ зависят от их источников. Источником продольного поля является амплитуда $S_{u}$, возвышающейся над плато $S_{0}$ части $\Gamma^{3}: \xi_{l} \sim S_{u}$ (см. "Среда мира"), а поперечного- "вращение" шума вокруг центра гдэна. Скорость вращения берётся равной единичной частоте $\Omega=1$ основного колебания гдэна, радиус $-1 / S$, 
где $S=S_{0}+S_{u} \approx S_{0}-$ полная амплитуда. Это даёт момент импульса $1 \cdot S \cdot(1 / S)=1=\hbar / 2$, соответствующий спину $1 / 2$, и ток амплитуды $j=S_{u} \Omega /\left(2 \pi / S_{0}\right)=S_{u} S_{0} / 2 \pi$. Поперечное поле оценивается из уравнения (32) без магнитного поля $\xi_{t} \sim \Omega \xi_{t} \sim \dot{\xi}_{t}=4 \pi j \sim 2 S_{u} S_{0} \sim S_{0} \xi_{l} \gg \xi_{l}$. Малость продольного поля $\xi_{l}$ позволяет им пренебречь и учитывать лишь поперечное поле $\xi_{t}$ как массивное вблизи гдэна, так и безмассовое вдали от него (поперечная электроволна). Продольным остаётся только кулоново поле электрозаряда.

Стационарность колебаний $\xi_{t}(R, t)$ означает отсутствие переноса $\ddot{\xi}_{t}(R, t)+\omega_{m}^{2} \xi_{t}(R, t)=0$, что приводит вблизи $\Gamma^{3}$ к уравнению $d_{R}\left[R^{-2} d_{R}\left(R^{2} \psi_{t} e^{-i \omega_{m}(R) t}\right)\right]=0$. Если пренебречь изменением частоты $\omega_{m}$ в пространстве, то $\psi_{t} \sim R^{-2}$.

Когда $\Gamma^{3}$ движется, $\xi$-поля перестают быть стационарными. Динамику поля $\xi$-колебаний в эфиронах удобно рассматривать используя уравнение для медленно меняющихся во времени амплитуд, вводя $\boldsymbol{\xi}(\mathbf{x}, t)=R e\left[\boldsymbol{\psi}(\mathbf{x}, t) e^{-i \omega_{m} t}\right]$. Тогда, пренебрегая $\ddot{\boldsymbol{\psi}}$, получим $\ddot{\boldsymbol{\xi}}=\left(\ddot{\boldsymbol{\psi}}-2 i \omega_{m} \dot{\boldsymbol{\psi}}-\omega_{m}^{2} \boldsymbol{\psi}\right) e^{-i \omega_{m} t} \approx-\left(2 i \omega_{m} \dot{\boldsymbol{\psi}}+\omega_{m}^{2} \boldsymbol{\psi}\right) e^{-i \omega_{m} t}$.

В нелинейной части (25), без учёта вторых гармоник (усреднение по периоду колебаний), запишем $\xi^{2}=|\psi|^{2} / 2$. Не учитывая также пространственное дифференцирование $\omega_{m}$, из (25) выведем амплитудное уравнение $-2 i \omega_{m} \dot{\boldsymbol{\psi}}=c^{2} \Delta_{t} \boldsymbol{\psi}+\omega_{\gamma}^{2}|\psi|^{2} \psi / 2$, из которого в отсутствие продольного поля $\operatorname{div} \boldsymbol{\psi}=0$ получим

$$
-i \dot{\boldsymbol{\psi}}=\Delta \boldsymbol{\psi} / 2 m+\omega_{\gamma}^{2}|\psi|^{2} \boldsymbol{\psi} / 2, \quad m=\omega_{m} / c^{2} .
$$

где $m$ - масса поперечных колебаний. Она вместе с $\omega_{m}$ меняется в пространстве вблизи $\Gamma^{3}$. Изменением скорости $c$, здесь можно пренебречь.

Если заменить переменную в пространстве массу на характерную постоянную $\bar{m}$, то получим нелинейное уравнение Шрёдингера (урШ) [5] с векторной переменной. Заменив её на скаляр, запишем урШ в обычном для нерелятивистской квантовой механике виде

$$
-i \dot{\psi}=\Delta \psi / 2 \bar{m}+\omega_{\gamma}^{2}|\psi|^{2} \psi / 2
$$

Вдали от $\Gamma^{3}$, где $\omega_{m}=0$, массивные колебания должны переходить в поперечные безмассовые $\xi$-волны.

В квантовой механике урШ описывает изменение волновой функции. Здесь это уравнение динамики амплитуды поперечных колебаний $\xi$-поля в ближайшей к гдэну области. Их тождественность возможна, если это 
поле составляет основу движения частицы. Тогда волновую функцию надо понимать как комплексную амплитуду массивных поперечных колебаний поляризации эфиронов вокруг гдэна, а массу частицы - как характерную массу $\bar{m}$ этих колебаний. В качестве неё можно например взять наибольшую массу поперечных $\xi$-колебаний $\bar{m}=\max \omega_{m}(R) / c^{2}$, достигаемую в ближайших к гдэну эфиронах.

Потенциальная энергия входит в урШ через нелинейность. Пусть амплитуда $\psi$ имеет две составляющие $\psi=\psi_{1}+\psi_{2}$. Тогда $\psi^{2}=\psi_{1}^{2}+$ $2 \psi_{1} \psi_{2}+\psi_{2}^{2}$. Нелинейность в урШ для $\psi_{1}$ разбивается на самодействие $\sim \psi_{1}^{2}$, взаимодействие $\sim 2 \psi_{1} \psi_{2}$ и воздействие $\sim \psi_{2}^{2}$. Если $\psi_{1} \ll \psi_{2}$, то остаётся только воздействие, имеющее обычный вид потенциала внешнего поля $U \psi_{1}, U=-\omega_{\gamma}^{2}\left|\psi_{2}\right|^{2} / 2$.

За квант $\xi$-колебаний берётся колебание с амплитудой $\psi=2$ в единицах амплитуды шума, чтобы его внешняя энергия была $\psi \omega_{m}=\hbar \omega_{m}$ при $\hbar=2$. Поскольку $|\psi| \ll 1$, то его энергия в одном эфироне не достигается, а распределена вокруг гдэна - квант коллективен.

При движении частицы переносится внутренняя энергия массивных $\xi$-колебаний. Её распределение пропорционально $|\psi|^{2}$ и подчинено уравнению непрерывности, которое следует из урШ (34). При нормировке на единицу образуется как-бы вероятностное распределение. Но частица неделима - её нельзя наблюдать по частям. Теперь $|\psi|^{2}$ представляется плотностью вероятности обнаружения частицы, как принято в квантовой механике, а не распределением энергии поперечных массивных $\xi$ колебаний внутри неё, как получено здесь.

В записи $\psi=|\psi| e^{i S}$ через модуль $|\psi|$ и фазу $S$ видно, что $|\psi|$ есть вещественная амплитуда массивных $\xi$-колебаний, а фаза волновой функции (действие частицы) - их относительное место.

Движение частицы есть перемещение её гдэна вместе с окружающим $\xi$-полем. Отдельный гдэн, двигаясь между эфиронами, притягивает противогдэн эфирона и отталкивает его гдэн. С противогдэном он объединяется в новый эфирон, а освободившийся гдэн заменяет его в двигающейся частице и поддерживает условия для существования массивного $\xi$-поля. Т. о. движение частицы есть перенос возмущения.

Движение частицы с любой скоростью можно рассматривать как распространение волнового пакета (или кванта волны), который берётся как набор близких по спектру волн. Они записываются на основе $(23)$ в виде направленного вдоль оси $l$ колебания на центральной частоте пакета $\omega(\mathbf{q})$ и его медленно меняющейся амплитуды 
$\xi(\mathbf{x}, t)=\operatorname{Re}\left[\psi(\mathbf{x}, t) e^{i \Phi}\right]=\psi e^{i \Phi} / 2+k c$,

$\Phi=-\omega t+\mathbf{q} \mathbf{x}, x=\{j, k, l\}, \mathbf{q}=\left\{q_{j}, q_{k}, q_{l}\right\}$,

где $k c$ означает комплексное сопряжение. Тогда, пренебрегая $\ddot{\psi}$,

$2 \ddot{\xi}=-\left(2 i \omega \dot{\psi}+\omega^{2} \psi\right) e^{i \Phi}+k c, \quad 2 \partial_{j}^{2} \xi=\partial_{j}^{2} \psi+2 i q_{j} \partial_{j} \psi-q_{j}^{2} \psi+k c$.

Так же для осей $k, l$. В нелинейной части рассматривается только самодействие и не учитываются гармоники $2 \Phi, 3 \Phi$.

$8 \xi^{3}=\psi^{3} e^{3 i \Phi}+3 \psi^{2} \psi^{*} e^{i \Phi}+\ldots=3|\psi|^{2} \psi e^{i \Phi}+k c$.

Теперь в (23) $-2 i \omega \dot{\psi}-\omega^{2} \psi+\omega_{m}^{2} \psi=$

$c^{2}\left[\partial_{j}^{2}+\partial_{k}^{2}+2 i\left(q_{j} \partial_{j}+q_{k} \partial_{k}\right)-q_{j}^{2}-q_{k}^{2}\right] \psi+c_{l}^{2}\left(\partial_{l}^{2}+2 i q_{l} \partial_{l}-q_{l}^{2}\right) \psi+3 \omega_{\gamma}^{2}|\psi|^{2} \psi / 8$.

После сокращения, вследствие диспур (27), остаётся

$-2 i \omega \dot{\psi}=\left[c^{2}\left(\partial_{j}^{2}+\partial_{k}^{2}\right)+c_{l}^{2} \partial_{l}^{2}+2 i \omega\left(V_{j} \partial_{j}+V_{k} \partial_{k}+V_{l} \partial_{l}\right)\right] \psi+3 \omega_{\gamma}^{2}|\psi|^{2} \psi / 8$,

где $\mathbf{V}=\left\{V_{j}, V_{k}, V_{l}\right\}-$ вектор групповой скорости. Отсюда в отсутствие

продольного поля следует $\partial_{l} \psi=0$ и

$-i d_{t} \psi=\left[\left(\partial_{j}^{2}+\partial_{k}^{2}\right) / 2 M+3 \omega_{\gamma}^{2}|\psi|^{2} / 8\right] \psi, d_{t} \psi=\dot{\psi}+\left(V_{j} \partial_{j}+V_{k} \partial_{k}\right) \psi$,

где $M=\omega / c^{2}-$ масса движущейся частицы.

Для перехода к векторной форме вводится вектор $\boldsymbol{\xi}(\mathbf{x}, t)=\operatorname{Re}\left[\boldsymbol{\psi}(\mathbf{x}, t) e^{i \Phi}\right]$ и пишется уравнение вектора амплитуды $\boldsymbol{\psi}$

$$
-i d_{t} \boldsymbol{\psi}=\left(\Delta / 2 M+3 \omega_{\gamma}^{2}|\psi|^{2} / 8\right) \boldsymbol{\psi}, \quad d_{t} \boldsymbol{\psi}=\dot{\boldsymbol{\psi}}+(\mathbf{V} \operatorname{grad}) \boldsymbol{\psi},
$$

где $\Delta$ - лапласиан. Это уравнение при $\omega_{m}=0, M=q / c, V_{j, k}=q_{j, k} / M$ применимо к описанию движения фотонов.

Масса движущихся квантов связана с их массой покоя дисперсионным уравнением

$\omega^{2}=\omega_{m}^{2}+c^{2} q^{2}, \quad V=c^{2} q / \omega, \quad \omega^{2}=\omega_{m}^{2}+\omega^{2} V^{2} / c^{2}, \quad \omega^{2}=\omega_{m}^{2} /\left(1-V^{2} / c^{2}\right)$, или при $\omega_{m} \neq 0: M^{2}=m^{2} /\left(1-V^{2} / c^{2}\right)$. Это известное соотношение масс механики Эйнштейна [2] получено здесь из рассмотрения волн в эфире. При $V \rightarrow 0$ имеем $\omega \rightarrow \omega_{m}, d \psi / d t=\dot{\psi}$ и (35) переходит в урШ (33) для движения частиц (квантов волн) с массой (покоя) $m$. Следовательно масса и движение частицы определяются окружающим их массивным $\xi$-полем, для которого гдэн является только затравкой.

Полученные результаты отличаются от принятого в современной квантовой механике толкования связи уравнений Шрёдингера и Клейна-ФокаГордона, при котором УрКФГ является релятивистским обобщением нерелятивистского урШ, применяемым к одной с ним переменной - волновой функции. УрКФГ (29) есть точное вещественное уравнение для вещественного вектора поляризации эфирона. УрШ (34) есть упрощённое комплексное уравнение для скалярной комплексной амплитуды этой 
поляризации. Релятивистским обобщением УрШ является (35), отличающееся от него полной производной по времени и массой движущихся квантов.

\section{Поле тяготения}

Деформация эфира имеет две причины. Это поляризация эфира $\xi$, которая меняет расстояние между эфиронами согласно (23), и чётная часть $U_{G}$ потенциала взаимодействия через двиг (11). Если поляризация эфиронов отсутствует, то остаётся $U_{G}$. При $U_{G}>0$ имеется всеобщее отталкивание, создающее условия для деформации нейтрального эфира и распространения в нём волн. Чтобы их исследовать берётся модель эфира как в "Поляризация среды". Неполяризованные эфироны расположены на невозмущённом расстоянии $r$ один от другого и образуют трёхмерную среду, за координатные оси $j, k, l$, которой берутся измеры (10).

Эфироны являются точками пространства. Вектор расстояния между ними $\mathbf{r}-$ его метрика. Деформация, как изменение этого расстояния на $\mathbf{h}$, меняет метрику, и её поле можно назвать метрическим.

Результатом действия потенциала отталкивания $U_{G}$ является уменьшение расстояния $h$ между эфиронами. Покажем это на примере. Пусть в невозмущённом эфире с расстоянием между эфиронами $r$ расположен дополнительный эфирон эо (или другой нейтральный объект) на расстоянии $r / 2$ от соседей. Он является центром симметрии и неподвижен. В равновесии остальные эфироны эl, где $l$ их номера, отсчитываемые от эо вдоль радиуса, смещаются на расстояние $a_{l}$. Ближайший к $\ni_{0}$ эфирон $\ni_{1}$ отталкивается до $r / 2+a_{1}>r / 2$, остальные - до $(l-1 / 2) r+a_{l}$. Расстояние между эфиронами меняется на $h_{l+1 / 2}=a_{l+1}-a_{l}<0: r_{l+1 / 2}=r+h_{l+1 / 2}$. Т. к. вдали от эо эфир не возмущён, то $h_{1 / 2}=-\sum_{l>1} h_{l+1 / 2}$. Относительное изменение метрики есть $\mu=(r+h)^{2} / r^{2} \approx 1+2 h / r$ при $h \ll r$.

При $h<0$ эфир сжимается. Таким же свойством обладает пространство с полем тяготения (тягполе) в теории гравитации Эйнштейна [3]. Их можно отождествить, если за потенциал тягполя взять изменение расстояния между эфиронами $h$ в некоторых единицах (см. далее). Тогда гравитация есть частный случай деформации эфира, а именно его сжатие под действием чётной части потенциала взаимодействия гдэнов через двиг. Это подтверждает метрический характер гравитации 
Эйнштейна и даёт ему обоснование.

Электроволны и частицы в поле тяготения. Их движение относительно эфира есть перенос возмущения $\xi$-поля, зависящий от расстояния $r$ между эфиронами. В сжатом эфире соседним эфиронам передаётся больше энергии, что увеличивает скорости переноса $c$ или $V-$ убыстряет электроволну или частицу. Скорость частицы $V=V(r+h(x))$. Ускорение $\dot{V}=V d_{x} V=V d_{r} V d_{x} h=-d_{x} u$, где $u=-d_{r}\left(V^{2}\right) h / 2$-потенциал действующего на частицу тягполя, который в соответствующих единицах измерения равен $h$. Для фотона (электроволны) $V$ меняем на $c$.

Уравнения поля тяготения зависят от используемой при их выводе модели эфира. Это связано с тем, что взаимодействие соседних эфиронов по поперечным и продольной осям отличаются качественно. Соседи по оси $l$ противодействуют росту возмущения и забирают себе часть его энергии, поддерживая волну переноса. А соседи по осям $j, k$ содействуют ему, приводя к перестройке решётки эфира в сторону увеличения пространственной симметрии, если только этому не препятствуют силы $\gamma$-взаимодействия. При $G$-отталкивании эфиронов наилучшие для равновесия условия имеет их сферически симметричное расположение все соседние эфироны находятся на сфере радиуса $r$ или близко к ней. Это может быть составленная из двух кубов решётка или непрерывное распределение по сфере, заменяющее решётку эфиронов.

Двойная решётка куба.

В решётке выделяется ось $l$ (продольная), вдоль которой эфироны смещаются при взаимодействии, и две поперечные оси $j, k$. Рассматриваемый эфирон эл помещается в начало отсчёта. На него действуют 14 эфиронов, расположенных на расстояние $r$ от него в двух решётках куба: 6 эфиронов (по два на каждой оси) составляют решётку 1. Ещё 8 эфиронов находятся в вершинах куба 2, грани которого пересекают оси на расстоянии $r / \sqrt{3}$.

Сначала рассмотрим решётку 1 . Координаты воздействующих эфиронов $\ni_{j k l}$ пишутся в единицах $r$ и порядке записи $j k l$. Продольное смещение обозначено $a_{j k l}$, при этом $a_{000} \equiv a$. Разность смещений $b_{j k l}=a_{j k l}-a$. Квадраты расстояний от э0 до э ${ }_{j k l}: \rho_{j k 0}^{2}=r^{2}+b_{j k 0}^{2}, \rho_{00 \pm 1}^{2}=\left(r \pm b_{00 \pm 1}\right)^{2}$.

Потенциал воздействия на эо есть $U_{1}(a)=\sum_{j k l} U_{j k l}$. Т. к. во взаимодействии участвуют гдэн и противогдэн эфирона, то потенциалы отталкивания (13) удваиваются: $U_{j k l}=2 G \exp \left\{-\rho_{j k l}^{2}\right\}$.

Воздействующая сила $f_{1}=-\partial_{a} U_{1}=\sum_{j k l} U_{j k l} \partial_{a} \rho_{j k l}^{2}$, где 
$\partial_{a} \rho_{j k 0}^{2}=-2 b_{j k 0}, \partial_{a} \rho_{00 \pm 1}^{2}=\mp 2 \rho_{00 \pm 1}$. Тогда

$f_{1}=-2\left[\sum_{j k} U_{j k 0} b_{j k 0}+U_{001} \rho_{001}-U_{00-1} \rho_{00-1}\right]$.

В линейном приближении при $|a| \ll r: U_{j k 0} b_{j k 0}=2 G e^{-r^{2}} b_{j k 0}$,

$U_{00 \pm 1} \rho_{00 \pm 1}=2 G \exp \left\{-r^{2} \mp 2 r b_{00 \pm 1}\right\}\left(r \pm b_{00 \pm 1}\right)=2 G e^{-r^{2}}\left(r \pm b_{00 \pm 1} \mp 2 r^{2} b_{00 \pm 1}\right)$,

$f_{1}=-4 G e^{-r^{2}}\left[\left(a_{100}+a_{-100}-2 a\right)+\left(a_{010}+a_{0-10}-2 a\right)\right.$

$\left.-\left(2 r^{2}-1\right)\left(a_{001}+a_{00-1}-2 a\right)\right]=-4 G e^{-r^{2}}\left[\left(\delta_{j}^{2}+\delta_{k}^{2}\right)-\left(2 r^{2}-1\right) \delta_{l}^{2}\right] a$.

Здесь введены разностные производные как в (19). Окончательно вклад эфиронов решётки 1 в действуюшую на э силу

$f_{1}=\left[-\sigma_{1}^{2}\left(\delta_{j}^{2}+\delta_{k}^{2}\right)+\sigma_{l 1}^{2} \delta_{l}^{2}\right] a, \quad \sigma_{1}^{2}=4 G e^{-r^{2}}, \sigma_{l 1}^{2}=\left(2 r^{2}-1\right) \sigma_{1}^{2}$.

Видно, что воздействие соседей по поперечным и продольной осям отличаются качественно - имеется поперечная неустойчивость, способная перестроить решётку 1 (если не учитывать влияние других сил).

Рассмотрим решётку 2. На э действуют 8 эфиронов $\ni_{J K L}$ в углах куба. Их координаты $J K L$ пишутся в единицах $\rho=r / \sqrt{3}$. Потенциал воздействия на выделенный эфирон есть $U_{2}(a)=\sum_{J K L} U_{J K L}$.

Квадраты расстояний от $\ni_{0}$ до $\ni_{J K L}$ в линейном приближении: $\rho_{J K \pm 1}^{2}=\left(\rho \pm b_{J K \pm 1}\right)^{2}+2 \rho^{2}=r^{2} \pm 2 \rho b_{J K \pm 1}$.

Потенциалы отталкивания эфиронов $U_{J K L}=2 G \exp \left\{-\rho_{J K L}^{2}\right\}$. $U_{2}=2 G e^{-r^{2}} \sum_{J, K= \pm 1}\left[\exp \left\{-2 \rho b_{J K 1}\right\}+\exp \left\{2 \rho b_{J K-1}\right\}\right]$.

Воздействующая сила $f_{2}=-\partial_{a} U_{2}$ :

$f_{2}=-4 G \rho e^{-r^{2}} \sum_{J, K= \pm 1}\left[\exp \left\{-2 \rho b_{J K 1}\right\}-\exp \left\{2 \rho b_{J K-1}\right\}\right]$

$=8 G \rho^{2} e^{-r^{2}} \sum_{J, K= \pm 1}\left(a_{J K 1}+a_{J K-1}-2 a\right)$.

Вводятся разностные производные как в (19). Тогда

$f_{2} e^{r^{2}} /\left(8 G \rho^{2}\right)=\sum_{J, K= \pm 1}\left(a_{J K 1}+a_{J K-1}-2 a_{J K 0}+2 a_{J K 0}\right)-8 a$

$=\left(\delta_{L}^{2}+2\right) \sum_{J, K= \pm 1} a_{J K 0}-8 a=\left(\delta_{L}^{2}+2\right)\left(\delta_{K}^{2}+2\right) \sum_{J= \pm 1} a_{J 00}-8 a$

$=\left(\delta_{L}^{2}+2\right)\left(\delta_{K}^{2}+2\right)\left(\delta_{J}^{2}+2\right) a-8 a=4\left(\delta_{L}^{2}+\delta_{K}^{2}+\delta_{J}^{2}\right)$,

если оставить только вторые производные.

Возвращаясь к расстоянию $r=\sqrt{3} \rho$ и решёточным переменным $j, k, l$ в единицах $r$ запишем $\delta_{J}^{2}=\delta_{j}^{2} \rho^{2} / r^{2}=\delta_{j}^{2} / 3, \ldots$ Тогда $f_{2}=\sigma_{2}^{2}\left(\delta_{j}^{2}+\delta_{k}^{2}+\delta_{l}^{2}\right) a$, где $\sigma_{2}^{2}=32 G r^{2} e^{-r^{2}} / 9=8 r^{2} \sigma_{1}^{2} / 9$.

Суммарная сила воздействия эфиронов двух решёток $f=f_{1}+f_{2}=\left[\sigma^{2}\left(\delta_{j}^{2}+\delta_{k}^{2}\right)+\sigma_{l}^{2} \delta_{l}^{2}\right] a$, где

$\sigma^{2}=\sigma_{2}^{2}-\sigma_{1}^{2}=4 G\left(8 r^{2} / 9-1\right) e^{-r^{2}}>0$, при $r^{2}>9 / 8$, a

$\sigma_{l}^{2}=\sigma_{2}^{2}+\sigma_{l 1}^{2}=4 G\left(26 r^{2} / 9-1\right) e^{-r^{2}}$.

Если не учитывать влияние эфиронов на поперечной плоскости $j, k$, то $\sigma^{2}=\sigma_{2}^{2}=32 G r^{2} e^{-r^{2}} / 9$.

Ускорение эфиронов $\ddot{a}=f_{1}+f_{2}$, а изменение расстояния между ними 
(потенциал поля тяготения) $\ddot{h}=\delta_{l} \ddot{a}:$ подчиняется уравнению

$$
\ddot{h}=\left[\sigma^{2}\left(\delta_{j}^{2}+\delta_{k}^{2}\right)+\sigma_{l}^{2} \delta_{l}^{2}\right] h,
$$

\section{Модель непрерывного распределения.}

На выделенный эфирон, имеющий смещение $a$ вдоль продольной оси $l$, воздействуют $N \sim 10 \div 15$ эфиронов, равномерно "размазанных"по сфере радиуса $r$ и смещённых на $a(\theta, \chi)$ вдоль той же оси. Т. к. во взаимодействии участвуют гдэн и противогдэн эфирона, то потенциалы отталкивания (13) удваиваются. Потенциал воздействия $U_{0}(a)=\int U d s$, где $U=g e^{-\rho^{2}}-$ его поверхностная плотность, $g=N G /\left(2 \pi r^{2}\right)$ - постоянная воздействия, $\rho^{2}=r^{2}+b^{2}+2 r b \cos \theta-$ расстояние между эфиронами, $b=a(\theta, \chi)-a \ll r, d s=r^{2} \sin \theta d \theta d \chi-$ элемент площади сферы, $\theta-$ угол с осью $l, \chi-$ угол в плоскости $j, k$, который отсчитывается от оси $j$.

Действующая на выделенный эфирон сила $f_{0}=-\partial_{a} U_{o}=\int f d s$, где $f=\partial_{b} U=-2(b+r \cos \theta) U$. Вводятся обозначения

$c=\cos \theta, \quad r_{c}=r c, \quad r_{s}=r \sin \theta, \quad h_{c}=r_{s} c, \quad h_{s}=r_{s} \sin \theta$. Тогда

$f_{0}=-2 g r^{2} e^{-r^{2}} \int_{-1}^{1} d c \int_{-\pi}^{\pi} d \chi\left(b+r_{c}\right) \exp \left\{-b^{2}-2 b r_{c}\right\}$.

Линейное приближение по $b / r$ даёт

$f_{0} e^{r^{2}} / 2 g r^{2}=-\int_{-1}^{1} d c \int_{-\pi}^{\pi} d \chi\left(b+r_{c}-2 b r_{c}^{2}\right)=\int_{-1}^{1} d c \int_{-\pi}^{\pi} d \chi\left(2 r_{c}^{2}-1\right)[a(\theta, \chi)-a]$

$=\int_{-1}^{1}\left(2 r_{c}^{2}-1\right) d c \int_{-\pi}^{\pi} a(\theta, \chi) d \chi-4 \pi\left(2 r^{2} / 3-1\right) a$.

Видно, что при $r_{c}^{2}=1 / 2$ сила $f$ меняет знак.

$$
\begin{aligned}
& \int_{-\pi}^{\pi} a(\theta, \chi) d \chi=\int_{-\pi / 2}^{\pi / 2}[a(\theta, \chi)+a(\theta, \pi-\chi)] d \chi=\int_{-\pi / 2}^{\pi / 2}\left[\left(h_{c} / r\right)^{2} \delta_{j}^{2}+2\right] a_{k}(\theta, \chi) d \chi \\
& =\int_{0}^{\pi / 2}\left[\left(h_{c} / r\right)^{2} \delta_{j}^{2}+2\right]\left[a_{k}(\theta, \chi)+a_{k}(\theta,-\chi)\right] d \chi \\
& =\int_{0}^{\pi / 2}\left[\left(h_{c} / r\right)^{2} \delta_{j}^{2}+2\right]\left[\left(h_{s} / r\right)^{2} \delta_{k}^{2}+2\right] d \chi a_{l}(\theta) \\
& =2 \int_{0}^{\pi / 2}\left[\left(h_{c} / r\right)^{2} \delta_{j}^{2}+\left(h_{s} / r\right)^{2} \delta_{k}^{2}+2\right] d \chi a_{l}(\theta)=\pi\left[\left(r_{s}^{2} / 2 r^{2}\right)\left(\delta_{j}^{2}+\delta_{k}^{2}\right)+2\right] a_{l}(\theta) .
\end{aligned}
$$

Здесь $a_{k}(\theta, \chi)=[a(\theta, \chi)+a(\theta, \pi-\chi)] / 2-$ среднее значение смещения на сфере, перенесённое на ось $k$, что необходимо для введения второй разностной производной $\delta_{j}^{2} \cdot a_{l}(\theta)=\left[a_{k}(\theta, \chi)+a_{k}(\theta,-\chi)\right] / 2-$ то же для $\delta_{k}^{2}$. Производные выше 2-го порядка не учитываются. 
Теперь, обозначая $\delta_{t}^{2}=\delta_{j}^{2}+\delta_{k}^{2}$, запишем

$f_{0} e^{r^{2}} / 2 g r^{2}+4 \pi\left(2 r^{2} / 3-1\right) a=\pi \int_{-1}^{1}\left(2 r_{c}^{2}-1\right)\left[\left(\sin ^{2} \theta \delta_{t}^{2} / 2+2\right] a_{l}(\theta) d c\right.$,

$=\pi \int_{0}^{1}\left(2 r_{c}^{2}-1\right)\left[\sin ^{2} \theta \delta_{t}^{2} / 2+2\right]\left[a_{l}(\theta)+a_{l}(\pi-\theta)\right] d c$

$=\pi a \int_{0}^{1}\left(2 r_{c}^{2}-1\right)\left[\sin ^{2} \theta \delta_{t}^{2} / 2+2\right]\left[\left(r_{c} / r\right)^{2} \delta_{l}^{2}+2\right] d c$

$=\pi a \int_{0}^{1}\left(2 r^{2} c^{2}-1\right)\left[\left(1-c^{2}\right) \delta_{t}^{2}+2 c^{2} \delta_{l}^{2}+4\right] d c$.

$=\pi a \int_{0}^{1}\left(2 r^{2} c^{2}-1\right)\left[\delta_{t}^{2}+4+\left(2 \delta_{l}^{2}-\delta_{t}^{2}\right) c^{2}\right] d c$

$=\pi a\left\{2 r^{2}\left[\left(\delta_{t}^{2}+4\right) / 3+\left(2 \delta_{l}^{2}-\delta_{t}^{2}\right) / 5\right]-\delta_{t}^{2}-4-\left(2 \delta_{l}^{2}-\delta_{t}^{2}\right) / 3\right\}$

$=\pi a\left[4\left(2 r^{2} / 3-1\right)+\left(4 r^{2} / 15-2 / 3\right) \delta_{t}^{2}+\left(4 r^{2} / 5-2 / 3\right) \delta_{l}^{2}\right]$.

Окончательно сила, действующая на выделенный эфирон

$f_{0}=\sigma_{3}^{2}\left(\delta_{j}^{2}+\delta_{k}^{2}\right)+\sigma_{l 3}^{2} \delta_{l}^{2}$, где

$\sigma_{3}^{2}=4 \pi g r^{2} e^{-r^{2}}\left(2 r^{2}-5\right) / 15>0$, при $r^{2}>5 / 2$, а

$\sigma_{l 3}^{2}=4 \pi g r^{2} e^{-r^{2}}\left(6 r^{2}-5\right) / 15, g=N G /\left(2 \pi r^{2}\right)$.

Уравнение потенциала тягполя совпадает с (36), если $\sigma=\sigma_{3}, \sigma_{l}=\sigma_{l 3}$.

Из-за противоположного влияния соседних эфиронов по поперечным и продольной осям в обоих моделях $\sigma^{2} \ll \sigma_{l}^{2}$.

Из-за слабости взаимодействия скорости переноса поля тяготе-

ния крайне малы по сравнению со скоростями $c$ и $c_{l}$ переноса поля поляризации (19): $\sigma^{2} / c^{2} \sim \sigma_{l}^{2} / c_{l}^{2} \sim G / \gamma$.

$\mathrm{B}$ приближении сплошной среды разностные производные в (36) заменяются на частные, а при переходе к векторной записи, смещение эфиронов вместе с потенциалом тягполя становятся векторами, подчиняющимися уравнению

$$
\ddot{\mathbf{h}}=-\sigma^{2} \operatorname{rot} \operatorname{rot} \mathbf{h}+\sigma_{l}^{2} \operatorname{grad} \operatorname{div} \mathbf{h},
$$

где координаты записаны в единицах расстояния между эфиронами.

Постоянное тягполе точечной массы в приближении сплошной среды находится из условия сферической симметрии. Неподвижное равновесие устанавливается когда напряжённость тягполя подчиняется геометрическому ослаблению $\sim R^{-2}$, или $h \sim 1 / R$, где $R-$ расстояние до тела. Это соответствует закону тяготения Ньютона.

Теперь поле тяготения складывается из трёх частей $\mathbf{h}=\mathbf{h}_{t}+\mathbf{h}_{l}+\mathbf{h}_{m}$, где $\mathbf{h}_{t}-$ поперечное поле, $\mathbf{h}_{l}-$ продольное, $\mathbf{h}_{m}-$ поле массивных тел. 
Их уравнения

$$
\begin{gathered}
\ddot{\mathbf{h}}_{t}=\sigma^{2} \Delta \mathbf{h}_{t}, \quad \ddot{\mathbf{h}}_{l}=\sigma_{l}^{2} \Delta \mathbf{h}_{l}, \quad \Delta h_{m}=4 \pi \rho_{m}, \\
\operatorname{div} \mathbf{h}_{t}=0, \quad \operatorname{rot} \mathbf{h}_{l}=\operatorname{rot} \mathbf{h}_{m}=0,
\end{gathered}
$$

где $\rho_{m}$ - объёмная плотность массы, $h_{m}=\left|\mathbf{h}_{m}\right|$.

Деформация эфира $\mathbf{h}$ имеет две части, обусловленные двумя причинами. Это $\mathbf{h}_{G}$, порождённая чётной частью $U_{G}$ потенциала взаимодействия гдэнов через двиг (11), и $\mathbf{h}_{\xi}$, нелинейно порождённая переменным электрополем $\boldsymbol{\xi}$ (23). Их соотношение для частиц (см. "Среда мира") $h_{G} \ll h_{\xi} \ll \xi: \quad h_{G} / h_{\xi} \sim U_{G} / U_{\gamma} \sim S_{u} / S_{0} \lesssim 10^{-50}, \quad h_{\xi} / \xi \sim \xi / r$.

Малость $h_{G}$ обуславливает известную слабость тяготения, которое только для огромного и электронейтрального множества частиц способно создавать заметные поля. При этом собственная скорость распростра-

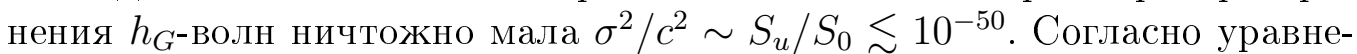
ниям $(23) h_{G}$-поле влияет на электроволну и может переноситься вместе с ней. Но оно много меньше собственного $h_{\xi}$-поля волны. А нелинейность их связи приводит к зависимости переносимого тягполя от параметров переносящей его электроволны, что затрудняет предсказание итога этого переноса. Тогда наблюдавшаяся в опытах [16] "гравитационная волна, сопровождаемая электроволной" , есть неотделимое от электроволны $h_{\xi}$-поле, которое будет очень непросто связать с гравитационными возмущениями, которые сопровождает эта электроволна.

Вещество является электронейтральным лишь для пространственных разрешений много больше размера атомов. На меньших масштабах всегда существуют сильные переменные электрополя. При движении вещества они вполне способны перестраивать поля тяготения приблизительно со скоростью света под текущее распределение вещества. Это не перенос, а именно нелинейная перестройка. Гравитоны, как переносчики только гравитации, здесь слабы - их можно не учитывать. В итоге остаётся обычное старое притяжение Ньютона, которое надо подправить на нелинейную перестройку электрополем. Уравнение (38) сокращается до

$$
\Delta h_{m}=4 \pi \rho_{m} .
$$

Очень сильное поле тяготения отражает значительное уменьшение расстояния между эфиронами, что должно вызвать фазовый переход эфира в состояние ГП-плазмы. В ней нет поля поляризации, следовательно отсутствуют электроволны и частицы. Эта область выглядит как чёрная дыра и очень даже возможно является ею. 


\section{Частицы}

Основными элементами нашего трёхмерного мира являются трёхместные гдэны $\Gamma^{3}, \Pi^{3}$ и одноместные пары $\nu_{j}$, которые составлены из одноместных гдэнов $\Gamma_{j}^{1}, \Pi_{j}^{1}$, соединённых обменными g-связями $(9,10)$. Места гармоник $\Gamma_{j}^{1}$ и $\Pi_{j}^{1}$ составляют их пространственные координаты.

Свойствами, совпадающими со свойствами одноместных гдэнов, обладают нижние кварки $[17,18]$, если их цвета $[19,20]$ отождествить с измерами. Тогда нижние кварки $d_{j}$ есть противогдэны $\Pi_{j}^{1}$, нижние антикварки - гдэны $\Gamma_{j}^{1}$, цвета кварков - измеры $j=1,2,3$, ответственные за размерности нашего пространства.

В адронах [21] кварки связаны глюонами [20] также, как $\Gamma_{j}^{1}$ и $\Pi_{j}^{1}$ связаны обменными g-связями $(9,10)$ в $\Gamma^{3}, \Pi^{3}$ и $\nu_{j}$. Следовательно глюоны есть g-связи. Но, в общем случае, они не кванты волн. Волна есть перенос через соседние точки. А в шуме многомерных g-связей каждый $\Gamma_{j}^{1}$ связан сразу со всеми $\Gamma_{i \neq j}^{1}$. Здесь нет волны. Трёхмерные гдэны являются исключением, в которых все $\Gamma_{j}^{1}$ оказываются соседями, что позволяет ввести представление о глюонах как квантах волн в "цветовом пространстве".

Обмены между $\Gamma_{j}^{1}$ и $\Gamma_{i}^{1}$ разных измеров $i \neq j$ дают 6 двухцветных глюонов. Обмены между $\Gamma_{j}^{1}$ и $\Pi_{j}^{1}$ одного измера дают 3 бесцветных глюона. Однако в квантовой хромодинамике принимаются лишь два из них, чтобы общее число сортов глюонов совпало с размерностью присоединённого представления группы $S U(3)$, к которой их привязывают. Считается, что третий бесцветный глюон не должен наблюдаться на опыте. В уравнениях же $(9,10)$ три обмена $\eta_{j}$ есть действующие в разных измерах независимые шумы. Они все должны учитываться. Не видно причин, чтобы отказываться от третьего бесцветного глюона.

Амплитуды $\Gamma^{3}$ и $\Pi^{3}$ втрое больше амплитуд $\Gamma^{1}$ и $\Pi^{1}$, что совпадает с соотношением зарядов электрона и кварков. Если электрические заряды частиц связать с амплитудами гдэнов (см. "Электрическое поле"), то напрашивается утверждение: Электрон $e^{-}$в своей основе $\left(O_{e}^{-}\right)$имеет противогдэн $O_{e}^{-}=\Pi^{3}$, а позитрон $e^{+}-$гдэн $O_{e}^{+}=\Gamma^{3}$. Следовательно они содержат кварки (нижние). Но кварки не дают им пространственного строения, т. к. места $\Gamma_{j}^{1}$ и $\Pi_{j}^{1}$ дают лишь точку пребывания. Только в этом смысле электрон и позитрон можно считать бесструктурными, как сейчас принято. Пространственное строение этих частиц определяется окружающими их дворами Д $\beth_{e}^{-}$и $Д_{e}^{+}$из $\xi$-полей, которые наделяют 
частицы массой.

Одноместные пары $\nu_{j}=\Gamma_{j}^{1} g g \Pi_{j}^{1}$ измеров $\mathrm{j}=1,2,3$, из-за их противофазных колебаний, представляют безамплитудные частицы (без заряда), но с внутренней энергией колебаний. K ним надо добавить 2-местные $\nu_{j} \nu_{k}, \nu_{j k}=\Gamma_{j}^{1} g \Pi_{j}^{1} g \Gamma_{k}^{1} g \Pi_{k}^{1} g, 3$-местные $\nu_{1} \nu_{2} \nu_{3}, \nu_{123}=\Gamma_{1}^{1} g \Pi_{1}^{1} g \Gamma_{2}^{1} g \Pi_{2}^{1} \Gamma_{3}^{1} g \Pi_{3}^{1} g$ и более длинные сочетания. Между ними должны быть переходы на основе реакций обмена $g$-связями, ведущие к установлению детального равновесия. Т. к. с ростом длины цепей растёт вероятность их распада, то наиболее устойчивы одноместные $\nu_{j}$ и их сочетания $\nu_{j} \nu_{k}, \nu_{1} \nu_{2} \nu_{3}$. Единственными частицами, на которые они похожи, являются нейтрино [22]. Тогда переходам между ними соответствуют нейтринные осцилляции [23].

Гдэн $\Gamma_{j}^{1}$ можно представить имеющим две свободные $g$-связи для взаимодействий с гдэнами других измеров. Если считать, что во взаимодействии с противогдэном своего измера участвуют обе связи, то это будет соответствовать представлениям о взаимодействиях посредством глюонов. Пары гдэнов разных измеров, связанные одной $g$-связью, имеют удвоенные амплитуды и две свободные связи. Они похожи на антикварки $\bar{d}_{j}$ с двойным зарядом. Это верхние кварки $u_{j}=g \Gamma_{j+1}^{1} g \Gamma_{j-1}^{1} g=\bar{d}_{j+1} \bar{d}_{j-1}$ и их антикварки $\bar{u}_{j}=g \prod_{j+1}^{1} g \Pi_{j-1}^{1} g=d_{j+1} d_{j-1}$.

Полученные сочетания одноместных гдэнов (кварков) вместе с фотонами $\gamma$, как квантами безмассовых $\xi$-волн (29-31), образуют первое поколение частиц:

$d_{j}=\Pi_{j}^{1}, \bar{d}_{j}=\Gamma_{j}^{1}, u_{j}=g \Gamma_{j+1}^{1} g \Gamma_{j-1}^{1} g, \bar{u}_{j}=g \Pi_{j+1}^{1} g \Pi_{j-1}^{1} g, \nu_{j}=\Gamma_{j}^{1} g g \Pi_{j}^{1}$, $e^{+-}=O_{e}^{+-}+Д_{e}^{+-}, O_{e}^{+}=\Gamma_{1}^{1} g \Gamma_{2}^{1} g \Gamma_{3}^{1} g, O_{e}^{-}=\Pi_{1}^{1} g \Pi_{2}^{1} g \Pi_{3}^{1} g, \gamma$.

Они самые лёгкие и не распадаются.

Нейтрино и основы электрона содержат наименьшее число связанных глюонами кварков. Эти $g$-связи первыми выделились из уменьшающегося шума. Их сила соответствует уровню шума при их рождении и является наибольшей среди остальных взаимодействий. Поэтому, как показано в квантовой хромодинамике (КХД), они практически не бывают свободными, объединяя кварки в замкнутые соединения. В $(9,10)$ это представлено парными корреляциями, дающими линейные $g$-связи. Но в КХД глюонные взаимодействия нелинейны и требуют учёта корреляций (8) более высокого порядка.

Следующими по порядку идут обмены между g-связями (глюонами). В $(9,10)$ добавляются произведения шумов, учитывающие эти 
обмены.

$$
\begin{aligned}
& S_{j}^{\Gamma}=\bar{S}_{j}^{\Gamma}+\epsilon_{j}+g S_{j}^{\Gamma} \sum_{i=1}^{3} \eta_{j i}\left(1+g_{1} \sum_{k, l=1}^{3} \tau_{i j, k l} \eta_{k l}\right) S_{i}^{\Gamma}, \quad \tau_{i j, k l}=-\tau_{k l, i j} . \\
& S_{j}^{\Gamma}=\bar{S}_{j}^{\Gamma}+\epsilon_{j}+g\left[\eta_{j}\left(1+g_{1} \tau_{j} \theta_{j}\right)+\theta_{j}\left(1-g_{1} \tau_{j} \eta_{j}\right)\right] S_{j}^{\Gamma} S_{j}^{\Pi}, \\
& S_{j}^{\Pi}=\bar{S}_{j}^{\Pi}+\epsilon_{j}-g\left[\eta_{j}\left(1+g_{1} \tau_{j} \theta_{j}\right)+\theta_{j}\left(1-g_{1} \tau_{j} \eta_{j}\right)\right] S_{j}^{\Gamma} S_{j}^{\Pi} .
\end{aligned}
$$

Здесь $\tau_{i j, k l}$ и $\tau_{j}$ - единичные шумы обмена между глюонами. При сильной нелинейности $g_{1} \sim g$ существует большая вероятность скоротечного разрыва $g$-связей с возможностью их переключения на другие кварки, имеющие в это время и в этом месте такие же временно разорванные $g$-связи.

Следующие поколения образуют более тяжёлые частицы с внутренним пространственным строением. Согласно имеющимся данным о строении частиц [24], все они включают в себя кварки, но имеют намного большие, чем у них, массы. Следовательно кварки составляют только их основу О, которую необходимо дополнить их окружением (двор Д).

Кварк-глюонные основы могут быть любой длины. Но с её ростом растёт вероятность разрыва какой-либо из внутренних $g$-связей и распада основы. В первом поколении представлены частицы с наименьшими замкнутыми основами, в которых случайные разрывы связей восстанавливаются в той же основе. Другие основы, имеющие бо́льшие длины, распадаются. Из них наименьшую длину имеют двуместные нейтрино $\nu_{i j}=\Pi_{i}^{1} g \Gamma_{i}^{1} g \Gamma_{j}^{1} g \Pi_{j}^{1} g=u \bar{u}$, спонтанно распадающиеся на одноместные. Также возможно существование трёх- и более местных нейтрино $\nu_{i j k} \ldots$ с поддержанием детального равновесия между ними. Примерами такого существования являются частицы без заряда, начиная с пиона $\pi^{0}$ и включая нейтрон.

Нейтральный пион имеет основу $O_{\pi}^{0}=u \bar{u}+d \bar{d}$, сводящуюся к паре $\Gamma^{3}, \Pi^{3}$ и дворы, содержащие нейтрино $\nu_{j}=d_{j} \bar{d}_{j}, \nu_{j k}$ и $\nu_{j k l}$. Его существование поддерживается реакциями обмена (40) между ними с перескоками $g$-связей. Он есть динамический и статистический объект в некотором нейтринном множестве, образующим нейтринную часть его двора, к которой ещё надо добавить $\xi$-двор (33), дающий пиону массу. Такую же основа имеет долгоживущий и более тяжёлый нейтрон $O_{n}=u d d=\nu_{i j}$. Он отличается от пиона только бо́льшим двором. Можно считать, что все нейтральные частицы имеет одинаковые основы из связанных 
полем поляризации гдэна и противогдэна $\Gamma^{3} \xi \Pi^{3}$ (они расположены на расстояние больше расстояния между эфиронами), и разные дворы, с которыми связаны их различия. Положительный пион $\pi^{+}$имеет основу $O_{\pi}^{+}=u \bar{d}=O_{e}^{+}$, совпадающую с основой позитрона. Такие же основы имеют все положительные частицы. И они разнятся только дворами. Отрицательные частицы имеют основу электрона $O_{e}^{-}$и разные дворы.

Среди частиц выделяется вечный протон. Его основа $O_{p}^{+}=u u d=$ $\Gamma_{1} g \Gamma_{2} g \Gamma_{3} g \Gamma_{1} g \Pi_{1} g=O_{e}^{+} \nu$ сводится к основе позитрона, связанной с нейтринным двором реакциями обмена. Это отражается в реакциях с участием протона, которые обязательно содержат нейтрино: $p \nu=e^{+} 2 \nu, p \nu=$ $n e^{+}, p e^{-}=n \nu, n=p e^{-} \nu$. Протон не статичен, а динамичен. Причину его устойчивости надо искать в строении двора и реакциях внутри него.

Имеются два взаимодействия гдэна с окружающей средой: $\gamma$ - потенциал (13), приводящий к $\xi$-полю и дающий массу частицам, а также связывающий гдэны $g$-обмен (40). Влияние первого преобладает в эфиpe, а второго - в нейтре. Обмен гдэна $\Gamma^{3}$ с нейтрино $\nu^{3}=\Gamma^{3} g g \Pi^{3}$ состоит из обмена гдэнами, который по сути ничего не меняет, и обмена гдэна с противогдэном, меняющим места гдэна и нейтрино, что приводит к случайным перемещениям гдэна в нейтре.

Глюонный $g$-обмен происходит при достаточной для него плотности числа нейтрино в пространстве, когда дворы гдэнов пересекаются. Если эта плотность меньшеет, то ближайшая к гдэну и связанная с ним часть нейтра сохраняет $g$-связь и начальную плотность. Образуется единая система, которая может быть устойчивой. Блуждания гдэна становятся ограниченными в пространстве. Повторные прохождения одного и того же места могут увеличивать расстояния между эфиронами до некоторого равновесного значения и тем придавать частице бо́льшую, чем у позитрона массу. Возможно так рождались протоны и нейтроны при уменьшении плотности числа нейтрино в расширяющейся вселенной.

Блуждания гдэна согласуются с опытами [25], из которых следует, что нуклоны имеют похожие пространственные распределения плотностей массы и заряда. Они убывают от центра размером $\sim 10^{-16}$ м до границы $\sim 10^{-15}$ м, что много больше расстояний между эфиронами $\sim 10^{-22}$ м. Дворы нуклонов существуют вместе с основами от их рождения в соответствующее время развития вселенной. Они должны нести в себе отпечаток среды того времени. Сейчас они являются переходной областью от основы через остаток среды его рождения к современной сре- 
де вселенной, скрывающийся за "пустым" пространством. Нейтринные дворы других тяжёлых частиц определяются условиями их рождения в соответствующих реакциях.

Все тяжёлые частицы описываются по схеме основа-двор. Их основы составлены из гдэнов и противогдэнов. Основа из нейтрино, если она возможна, считается отсутствующей, т. к. не отличается от нейтринной части двора. Вводить тяжёлые кварки для объяснения тяжёлых частиц нет необходимости. Достаточно трёх кварков (нижних) и их антикварков. Различия частиц связаны со строениями дворов. Бозоны слабого взаимодействия и мезоны не выделяются из других частиц, кроме своего участия в образовании ядерной материи. Строение мюонов и таонов не имеет качественных отличий от строения адронов.

В частицах с дворами различие фермионов и бозонов условно. Строго фермионами являются только гдэны вследствие их $\gamma$-отталкивания (13), не позволяющего им быть в одном месте. Бозонами являются только кванты волн, т. к. они свободно проходят один сквозь другой. Частицы, имеющие в своём составе гдэны и $\xi$-поля, не могут быть только фермионами или бозонами.

Спин. Основное колебание с единичной частотой в размытом шумом двуместном или трёхместном гдэне $(6,7)$ можно представить как вращение шума со скоростью $\dot{\varphi}=1$ в пространстве мест. С ним связан момент вращения $J$ вокруг оси, проходящей через его центр. Если амплитуда гдэна $S$, то радиус вращения $\sim 1 / S$ и $J \sim S \dot{\varphi} \cdot(1 / S) \sim 1=\hbar / 2$. Т. о. размытое шумом основное колебание гдэна является причиной спина $1 / 2$ во всех частицах, кроме кварков из-за их одноместности. Другие значения спина являются производными от него.

Наличие спина $1 / 2$ и свойство быть фермионом имеют разные причины. $\mathrm{K}$ невозможности двум одинаковым частицам быть в одном состоянии приводит их $\gamma$-отталкивание (13). Оно присуще и нейтральным частицам, составленным из нейтрино. Не имея заряда в среднем, они обладают флуктуирующим зарядом вследствие обменов между образующими их гдэном и противогдэном $(10,40)$. Тогда все основы частиц фермионы и имеют спин $1 / 2$.

О бозонах Намбу-Голдстоуна и Хиггса. Для их существования требуется отрицательный квадрат массы $\omega_{m}^{2}<0$ и очень большой его нелинейный рост с ростом величины поля Хиггса. Однако при $\omega_{m}^{2}<0$ ГП-пары распадаются - эфир переходит в плазму из трёхместных гдэнов и противогдэнов, в которой невозможна поляризация и соответству- 
ющие ей массивные $\xi$-поля. Сомнительной представляется и столь большая нелинейность. Поэтому сомнительно существование указанных бозонов. Более естественно предложить другой источник масс частиц.

Энергия-масса покоя массивной волны (33) $m c^{2}=\omega_{m}$ есть импульс (внешэн) колебания гдэна и противогдэна в ГП-паре с учётом влияния соседних пар, а $\omega_{m}^{2}$ - его внутэн $(26)$. Он складывается из внутэна $\omega_{\gamma}^{2}$ колебания свободного маятника ГП (15) и уменьшающего его влияния соседей, выраженного как внутэн переноса $4 c^{2}+2 c_{l}^{2}$. При большом переносе вся энергия маятника ГП может уноситься из ГП-пары, образуя волну с нулевой массой покоя. При малом переносе расходуется только часть этой энергии, и волна остаётся массивной. Если массы частиц определяются массивным $\xi$-полем, окружающим их основы, то все они связаны с частотой $\omega_{\gamma}$ и выражаются через неё. Т. к. $\omega_{\gamma}$ есть наибольшая частота колебаний гдэнов в ГП-парах, то связанная с нею масса кванта массивных волн поляризации тоже наибольшая. Но она недостижима из-за отсутствия пар вне ГП-среды. Т. о. все массы частиц связаны с частотой $\omega_{\gamma}$ линейных колебаний маятника ГП.

Относительность движения. Все частицы, возможно кроме нейтрино, получают массу от двора массивных стационарных колебаний и движутся вместе с ним, как возбуждения эфира. Связанная с эфиром система отсчёта абсолютна. Но наблюдатель или прибор могут двигаться только с частицами, из которых состоят тела. В сопровождающей их системе отсчёта групповая скорость массивной волны вместе с импульсом кванта (волновым вектором) становятся нулевыми, а частота и масса кванта уменьшаются до значений состояния покоя. Скорости и импульсы квантов других частиц, включая фотоны, отсчитываются от нуля. Т. е. скорости частиц всегда меньше предельной скорости переноса (света), а скорость фотона равна ей. Следовательно в любой движущейся с наблюдателем системе отсчёта скорость света постоянна и все физические процессы протекают одинаково. Абсолютность эфира скрывается за относительностью физических систем отсчёта.

Оценка величины основной частоты. За надшумную энергию гдэна берётся общепринятое значение энергии кварка $\sim 10^{-1}$ Эв, которое считается порядка энергии шума. Но шум возвышается над плато основания (см. "Среда мира"), которое на $\sim 46$ порядков его мощнее. Тогда энергия гдэна $\hbar \Omega=7 \cdot 10^{-16} \Omega \sim 10^{45}$ Эв, что даёт оценку основной частоты $\omega \sim 10^{60}$ Гц. 


\section{Заключение. Итоги}

В физике существует представление о "теории всего" как цели, к которой надо стремиться через обобщение и объединение уже известных теорий взаимодействий и строения материи. Предлагаемая здесь работа также сделана ради этой цели с надеждой на её достижение, но другим путём. Вместо дальнейшего отвлечения и усложнения математических орудий исследований, упор сделан на поиске относительно простого основания физики.

Такой выбор требует соответствующего ему названия предлагаемой здесь теории. Оно должно быть похоже на общепринятое название "теория всего" и отличаться от него. Такими свойствами обладает выражение панория. Оно составлено из двух древнегреческих слов: "пан" - всё и "теория" от которого взята вторая половина названия. В проведённом исследовании только начато вхождение в обширную область этой теории. На основе простейших доступных моделей ставятся вехи панории с надеждой на их дальнейшую более полную разработку.

Начало работе полагает утверждение о всеобщности энергии, понимаемой как способность совершать изменения (работу), т. е. быть изменением, которое передаётся при обмене (взаимодействии). Изменение представляется через переменные действие-длительность, что даёт наиболее общее его выражение. Энергия есть его скорость. Мерой длительности выступает время. Так понимаемое изменение принимается за основу всего сущего - мир изменений (мен).

Стационарные повторы выражаются через гармоники. Гармоника, кроме частоты и амплитуды, характеризуется "начальной фазой", которая есть её относительное место. Оно подобно месту тел и наделяет множество гармоник свойством одномерного пространства. Гармоника действие-энергия, с помощью которой строится теория, названа гдэн, а противофазная ей - противогдэн.

В гдэне имеется две энергии . Внешняя энергия, которая есть импульс колебания, способна передаваться другим гармоникам при обмене. Внутренняя энергия поддерживает колебание. Её механизм выделяется особо, как двигатель колебания, и назван двиг.

После выделения гармоник одной частоты, остальные гармоники с меньшими частотами задают её переменную среднюю линию, а с бо́льшими - модуляцию колебаний, или шум на этой частоте. Теперь в гармониках любой частоты представлен весь мен. Шум берётся за основное состоя- 
ние нашего мира. Он вне пространства, но содержит в себе его зачатки, включая число измерений.

Устойчивая структура, необходимая для существования мира, образуется из надшумных гдэнов. Предполагается, что надшумные гдэны возникали при ослаблении шума с сохранением его флуктуаций. Этот процесс сопровождался образованием корреляций между прежде независимыми шумами разных измерений и привёл к существованию многоместных гдэнов. Элементами мира стали трёхместные гдэны $\Gamma^{3}$ и противогдэны $\Pi^{3}$, составленные из одноместных гдэнов $\Gamma_{j}^{1}$ и противогдэнов $\Pi_{j}^{1}$ разных измерений $\mathrm{j}=1,2,3$ а также одноместные пары $\Gamma_{j}^{1} \Pi_{j}^{1}$ каждого измерения. Гдэны $\Gamma_{j}^{1}$ тождественны нижним кваркам $d_{j}-$ это единственные кварки, из которых составлены все частицы. Связи между измерениями известны как глюоны.

Кроме взаимодействия между одноместными гдэнами существует взаимодействие гдэнов через двиг. Его потенциал состоит из нечётной и чётной по амплитудам гдэнов частей. Нечётная часть ( $\gamma$-взаимодействие) обуславливает появление поля поляризации, включая известное электрополе. Чётная часть становится причиной поля тяготения.

Трёхмерное пространство нашего мира есть пространство мест гдэнов. Оно ограниченно, замкнуто на себе и имеет материальное основание. Среда нашего мира состоит из ослабленного шума, надшумного основания и возвышающихся над ним частей надшумных гдэнов.

Надшумные части одноместных гдэнов (кварки) собраны в элементы среды и отдельные частицы. Связанные $\gamma$-взаимодействием ГП-пары составили твёрдую среду ГП-твердь, а несвязанные - плазмообразную ГП-плазма. Переходная между ними среда есть основа нашего мира. Как носитель электрополей она получила давно предложенное для такой среды название - эфир (Э). Он состоит из эфиронов (ГП-пар) с определённым расстоянием между ними. Другая часть среды составлена из нейтрино и названа нейтр $(N)$. Их совокупность - эфней $(Э N)$. Возможно эфир есть 3-мерная поверхность в 4-мерной среде, разделяющая два её состояния: ГП-плазму и ГП-твердь.

Предложена модель среды нашего мира. Найдены уравнения динамики её поляризации и дисперсионное. Волны имеют поперечную и продольную составляющие. Но продольная волна намного слабее поперечной из-за более слабого источника внутри частиц и ей можно пренебречь.

Безмассовая поперечная волна поляризации описывается уравнениями Максвелла для электромагнитных волн в пустоте. Вместе с урав- 
нением кулоновой части постоянного поля поляризации вокруг частищ образуется система уравнений Максвелла. Это позволяет сделать вывод, что электрическое поле есть поляризация эфиронов, а магнитное поле - его изменение. Электромагнитная волна есть поперечная волна поляризации эфира.

Вокруг гдэнов в эфире существует $\xi$-поле поляризации эфиронов, которое входит в состав частиц. Вблизи них расположены стационарное поперечное массивное $\xi$-поле, дающее частицам массу. Далее расположено кулоново электрополе.

Для движущейся частицы динамика $\xi$-поля описывается уравнением Клейна-Фока-Гордона. После перехода к амплитудному описанию оно заменяется на нелинейное уравнение Шрёдингера. Показано, что волновая функция есть амплитуда колебаний гдэнов в эфироне. Уравнение для волнового пакета массивной волны поляризации даёт его движение и строение, учитывающее самодействие волны. Делается вывод о полевой природе массы и движения тел.

Поле тяготения (тягполе) обусловлено чётной по амплитуде частью потенциала взаимодействия гдэнов через двиг и является одной из частей поля деформации (метрики) эфира. Потенциал тягполя описывается изменением расстояния между эфиронами. Показано, как тягполе влияет на распространение электроволны и частиц.

Волны тяготения существуют, но из-за своих ничтожно малых величины и скорости их влияние столь же мало. Остаётся только поле притяжения Ньютона, которое при движении тел надо подправить на его нелинейную перестройку электрополем.

Трёхместные гдэны $\Gamma^{3}$ и противогдэны $\Pi^{3}$ составляют основу позитрона и электрона. Пары одноместных гдэнов $\Gamma_{j} \Pi_{j}$ есть электронные нейтрино. Верхние кварки $u_{j}$ составлены из пар гдэнов или противогдэнов $u_{j}=\bar{d}_{j+1} \bar{d}_{j-1}, \bar{u}_{j}=d_{j+1} d_{j-1}$. Все, кроме фотона, частицы первого поколения построены из кварков. В строение позитрона и электрона надо включить дворы из полей поляризации эфиронов.

Все имеющие массу частицы, кроме нейтрино, содержат лёгкие основы и окружающие их массивные дворы, которые состоят из полей поляризации эфиронов и нейтринной части. Дворы долгоживущих протонов сохраняют отпечаток среды вселенной, бывшей при их рождении.

Частицы вместе с окружающим их полем являются переносимыми возбуждениями эфира. Они имеют постоянное строение и не обладают постоянным составом. Для нашей вселенной эфир абсолютен, но прояв- 
ляется через относительность движения и строение мира, невозможное без его существования.

Таким образом показано, что теории современной физики могут быть выведены из представления всего существующего в виде одномерного изменения действие-длительность.

Пан А. Н. 2020. Вехи панории. Действие, как основа материи, пространства, мира. PREPRINTS.RU. https://doi.org/10.24108/preprints-3111976

Почта: panory@bk.ru

\section{Список литературы}

[1] E. Whitteker, A history of the theories of aether and electricity. Thomas Nelson and sons Ltd. (1910, 1953). Э. Уиттекер, История теории эфира и электричества. РХД, ИКИ, Москва, Ижевск $(2001,2004)$.

[2] A. Einstein, Ann.phis., 17, 891 (1905). А.Эйнштейн, Собрание научных трудов, 1, 7. Наука, Москва. (1965).

[3] A. Einstein, Ann.phis., 49, 769 (1916). А.Эйнштейн, Собрание научных трудов, 1, 452. Наука, Москва. (1965).

[4] W. Heisenberg, Zs. phys., 33, 879 (1925).

[5] E. Schrödinger, Annalen der Physik, 79, 361, 489 (1926).

[6] A. Einstein, Ather und Relativitätstheorie. Verlag von Julius Springer. (1920). А.Эйнштейн, Собрание научных трудов, 1, 682. Наука, Москва. (1965).

[7] P. A. M. Dirac, Proc. R. Soc. Lond. A., 126, 360 (1930).

[8] А.М. Ляпунов, Записки Академии наук по физ.-мат. отделению, серия 8. 12-5, 1-24. 1901. Собрание сочинений 1, 161. Москва: Издательство АН СССР, 1954.

[9] В.С. Пугачёв, Теория случайных функиий. Госиздатфизматлит, Москва (1950). 
[10] Королюк В.С. и др., Справочник по теории вероятности и математической статистике. Наука, Москва (1985).

[11] Van Dyck R. S. Jr., Schwinberg P. B., Dehmelt H. G.,. Phys. Rev. Lett. 59, 26. Х. Демельт, УФН. 160, 12, 129 (1990).

[12] A. Einstein, Sitzungsber.preuss. Akad. Wiss., 1, 142 (1917). А.Эйнштейн, Собрание научных трудов, 1, 601. Наука, Москва. (1965).

[13] O. Klein, Zeitschrift für physik 37, 895 (1926).

[14] V. Fock, Zeitschrift für physik 39, 226 (1926). В. А. Фок, УФН, 180, 874 (2010).

[15] W. Gordon, Zeitschrift für physik. 40, 117 (1926).

[16] B. P. Abbott et al. (LIGO and Virgo Scientific Collaborations), Phys. Rev. Lett. 116, 061102 (2016).

[17] M. A Gell-Mann, Physics Letters, 8, 214 (1964).

[18] Gн. Zweig, CERN Report № 8182/TH, 401 (1964).

[19] N. Bogolubov, B. Struminsky, A. Tavkhelidze, JINR Preprint D-1968, Dubna (1965).

[20] M. Y. Han, Y. Nambu, Phys. Rev. 139, 1006 (1965).

[21] L. B. Okun, in Proceedings of Intern. Conf. on High-energy Physics, CERN (1962), 845.

[22] W.E. Pauly, in Theoretical physics in the twentieth century. Ed. by M. Fierz and. V. F. Weisskopf, Interscience Publishers Inc., Ltd. New York, London (1960). Теоретическая физика XX в.. Иностранная литература, Москва (1962), 386.

[23] Б. Понтекорво, ЖЭТФ, 33, 549 (1957).

[24] Элементарные частицы. "Физ. энцикл. словарь". Сов. энциклопедия, Москва (1984), 896.

[25] R.Hofstadter, Science, 136, 1013 (1962). Р. Хофштадтер, УФН, 81, 185 (1963). 